\title{
Cross-Sectional Association of Adiponectin with Sociodemographic and Nutrition-Related Factors in Mexican Students from the Los Altos, Jalisco Region
}

\author{
Yesica Sughey González-Torres \\ University of Guadalajara \\ Napoleón González-Silva \\ University of Guadalajara \\ Angel Perez-Reyes \\ University of Guadalajara \\ Luis F Guzman \\ National Genetic Resources Center \\ Trinidad Garcia-Iglesias \\ University of Guadalajara \\ Christian M Rodriguez-Razon \\ University of Guadalajara \\ Zuamí Villagrán-de la Mora \\ University of Guadalajara
}

\section{Edgar A Rivera-Leon}

University of Guadalajara

Patricia N Vargas-Becerra

University of Guadalajara

\section{Sergio Sanchez-Enriquez}

University of Guadalajara

Maritza Roxana Garcia-Garcia ( $\sim$ maritza.garcia@edu.uag.mx )

Universidad Autonoma de Guadalajara https://orcid.org/0000-0002-2996-8264

\section{Research article}

Keywords: Adiponectin, Nutritional status, Body composition, Obesity, Underweight, Metabolism, Mexican traditions

Posted Date: July 2nd, 2020

DOI: https://doi.org/10.21203/rs.3.rs-39572/v1

License: (c) (i) This work is licensed under a Creative Commons Attribution 4.0 International License. Read Full License 


\section{Abstract \\ Background}

Adiponectin is a biomarker useful for the early diagnosis of obesity-related comorbidities. The Mexican population has one of the highest obesity rates worldwide. The purpose of this study was to determine whether an association exists between adiponectin and certain sociodemographic and nutrition-related factors in young adults. This is perhaps one of only a few studies focused in the Los Altos, Jalisco area, a center for religious and cultural traditions in Mexico.

\section{Methods}

A cross-sectional analysis was conducted from January to November 2019 at the Los Altos University Center. A total of 173 student volunteers participating in the study provided voluntary signed, written consent forms.

Sociodemographic data were collected. Biochemical and nutritional assessments were realized after eight hours of fasting. Dietary questionnaires were analyzed using Nutrikcal ${ }^{\circledR}$ software. Total serum adiponectin levels were quantified by enzyme-linked immunosorbent assay. Correlations and multivariate models were computerized by SPSSv20. This research project was approved and registered by the Research and Bioethics Committee of the Los Altos University Center, Mexico.

\section{Results}

According to body mass index (BMI), the incidence of underweight participants was found to be $10.4 \%$, whereas that for overweight and obese patients combined was found to be $23.1 \%$. A positive correlation of serum adiponectin levels was found in the following categories: female gender $(r=0.353, p=0.01)$; high-density lipoprotein cholesterol $(\mathrm{HDL}-\mathrm{c})(r=0.487, \mathrm{p}=0.01)$; and fruit intake $(r=0.204, \mathrm{p}=0.031)$. Multivariate analysis showed that $41.6 \%$ of adiponectin levels measured could be explained by gender, glucose, HDL-c, sodium and ascorbic acid consumption $\left(r^{2}=0.416, \mathrm{p}<0.01\right)$.

\section{Conclusions}

In accordance with the results, the incidence of underweight participants was greater than that expected as contrasted by national reports generated in Mexico. In addition, adiponectin level was found to vary with gender and nutrition-related factors. Further studies should be performed in this population to assess the role of religious and Mexican cultural traditions on health status in an effort to prevent undernutrition and obesity-related morbidity and mortality.

\section{Introduction}

Obesity is described as a nutrition-related disease recognized as a potential risk factor for the development of comorbidities including insulin resistance, hypertension, atherosclerosis, and hyperlipemia [1, 2]. According to the Organization for Economic Cooperation and Development (OECD), the population of Mexico has one of the highest obesity rates worldwide, placing the country second in the world with the most adult obesity [3]. Currently, based on the National Survey on Health and Nutrition (ENSANUT-2018), the prevalence of obesity in Mexico is $36.1 \%$ in the 
adult population, $14.6 \%$ in adolescents (12-19 years old), and $17.5 \%$ in children (5-11 years old). This is an alarming statistic with clear negative impact on public health given the principal causes of adult mortality in Mexico are nutrition-related, chronic pathologies such as type 2 diabetes, cardiovascular disease and cancer [4, 5]. Although obesity is a preventable disease, it has grown faster in the last few decades. Obesity is considered a multifactorial disease caused by lifestyle choices, greater availability of processed foods, sedentarism and other factors related to a so-called obesogenic environment [6]. Mexico, a country with wide demographic and territorial diversity, has experienced economic growth and urbanization within its population tending toward urban area concentration and rural dispersion. In addition, certain rural areas in Mexico maintain long-standing cultural traditions, particularly as these relate to dietary practices, may provide a unique insight as to the obesity problem faced in the country. In this context, the Los Altos Jalisco region, located in the central, western part of Mexico and characterized by a population with a long tradition of religious devotion since the seventeenth century, having as its religious center the second most-visited shrine in Mexico and Latin America, was selected for this study. In addition to its characteristic cultural traditions, the Los Altos region is known as a hub for religious tourism, farming and gastronomy, as well as other economic activities including the sale of religious articles, jewelry, textiles, livestock, poultry, and confections [7].

Because the population of Mexico experiences extremely diverse living conditions, it is believed immediate action must be taken in order to address the obesity problem in the country. Successfully addressing this problem in Mexico will diminish chronic, non-communicable diseases in children, adolescents, and adults, as well as decrease the social and economic costs associated with obesity [8]. Detection of biomarkers that enhance earlier diagnosis of obesityrelated comorbidities and form a cost-effective strategy for disease prevention is essential. In this regard, adiponectin is an ideal biomarker. Adiponectin is an adipokine primarily encountered as a $30 \mathrm{kDa}$ adipocyte complement-related protein (Acrp30) and a $28 \mathrm{kDa}$ gelatin-binding protein (Gbp28). Adiponectin, secreted mainly by adipocytes, has been extensively studied as one of the most abundant circulating proteins in humans ( 3 to $30 \mu \mathrm{g} / \mathrm{mL}$ in plasmatic levels). However, paradoxically, its levels decrease with excessive adipose tissue deposition (obesity) and with metabolic abnormalities such as insulin resistance and type 2 diabetes [9]. Adiponectin plays a key role in energy metabolism regulation by virtue of its anti-inflammatory, insulin-sensitizing and antiatherogenic properties [10-12]. Through activation of its two main receptors, AdipoR1 and AdipoR2, adiponectin modulates inflammation through the inhibition of nuclear factor KB (NF-kB), and regulates carbohydrate and lipid metabolism, stimulating glucose uptake in skeletal muscle and fatty acid oxidation in muscle and liver tissue [13-15]. Hence, adiponectin levels are now considered an effective biomarker in the prevention of obesity-related diseases, including type 2 diabetes, cardiovascular disease, and cancer [16-18].

Modulation of human adiponectin circulating levels has been researched in previous studies. Some studies have found that the expression of adiponectin is regulated by food consumption [19-21]. Other studies have shown a relationship between elevated adiponectin expession and a Mediterranean-type diet which includes generous portions of vegetable protein, polyunsaturated fatty acids, high-fiber whole grains, fruits and dairy products [22, 23]. Despite these findings, promoting a Mediterranean-type diet in Mexico may not be practical given its cultural attributes and its ready accessibility to food products constituting the traditional Mexican diet, markedly diverse from those of a Mediterranean-type diet [24]. Therefore, it is believed different results will exist in adiponectin studies conducted as between diverse geographic populations with differing diets. Therefore, in this study, the objective was to determine whether an association exists between serum adiponectin levels and sociodemographic and nutritionrelated factors in a young student population from the Los Altos, Jalisco region of Mexico. Most studies have evaluated adiponectin levels in association with western diets and obesogenic environmental factors in urbanized populations. This study takes a more novel approach focusing rather on a population from a traditional, rural Mexican area that typically enjoys a traditional Mexican diet. Hence, it was hypothesized potentially different 
adiponectin level associations would be found in this population. The intent of this study, perhaps the first of its kind, is to identify modifiable and non-modifiable factors associated with serum adiponectin levels in order to plan future health promotion strategies and minimize nutrition-related diseases in this particular population which, according to our knowledge, has preserved Mexican traditions.

\section{Methods}

\section{Study design and participants}

A cross-sectional study design was conducted on a student-based, recruited sample. Data collection and analyses were performed in conjunction with the Biosciences and the Nutritional Assessment Laboratories of the Los Altos University Center, University of Guadalajara, Mexico from January to November, 2019. The student volunteers participating in the study provided voluntarily signed, written consent forms. Eligible subjects were apparently healthy students from the Los Altos Jalisco region aged 18-25 years old. For this study, criteria for non-inclusion were consumption of lipid-lowering or body weight reduction medications, previous diagnosis of certain pathological conditions (diabetes mellitus, diseases of the kidneys and liver), as well as a physiological state of pregnancy or lactation in the case of female volunteers. This research project was peer-evaluated and approved by the Health Sciences Department from the Los Altos University Center as part of a grant application dated October 30th, 2017 and was approved and registered by the Research and Bioethics Committee of the Los Altos University Center, Mexico [CUA/CEI/027/2019]. The study was conducted according to ethical principles for medical research involving human subjects.

\section{Nutritional measurement}

The nutritional status of the participants was evaluated in accordance with the assessment step of the Nutrition Care Process (NCP) proposed by the Academy of Nutrition and Dietetics [25]. To avoid bias in data collection, anthropometric measurements were taken by nutrition and dietetic practitioners (Y.S.G.T. nutritionist) certified by the International Society for the Advancement of Kinanthropometry (ISAK). Body composition was analyzed by bioimpedance body analyzer equipment (TBP-300 Tanita Inc®) with participants in light clothing and after an eighthour fast. The body height measurement was taken utilizing a Frankfort plane and portable stadiometer (Seca ${ }^{\circledR}$ $\mathrm{GmbH} \&$ Co. KG company). Waist circumference was taken with Seca ${ }^{\circledR}$ ergonomic circumference measuring tape as directed by the protocols of anthropometric measurements by the ISAK Society [26]. The body mass index (BMI) was calculated using the Quetelet index formula $\left(\mathrm{Kg} / \mathrm{m}^{2}\right)$. The BMI of each participant was then classified in underweight, normal weight, overweight and obesity categories as delineated by the World Health Organization (WHO) [27]. The serum biochemical parameters were evaluated immediately after blood sample collection from the antecubital vein with participants having been in an eight-hour fast. Subsequently, the remainder of the serum was aliquoted in $600 \mu \mathrm{L}$ microtubes and quickly frozen at $-80^{\circ} \mathrm{C}$ for subsequent immunoassay assessment. In order to obtain biochemical data, the samples were processed in COBAS C-111 equipment for clinical chemistry analysis (Roche Diagnostics ${ }^{\circledR}$ ) using Cobas Reagent kits provided by Roche ${ }^{\circledR}$. Daily analytical sessions, including calibration and validation of the sample biochemical results, were done using a control reagent system. The Friedewal formula [total cholesterol minus high-density cholesterol (HDL-c) minus very-low density cholesterol (VLDL-c)] was used to estimate low-density lipoprotein (LDL-c) levels [28]. To complete a clinical and food-related history, registered nutritionists interviewed each participant. The dietary assessment was obtained from each interviewed participant through a 24-hour dietary recall registry that comprised recording of all food and beverages consumed within the previous 24 hours [29]. In addition, a prospective food intake questionnaire was given to assess meals and beverages consumed over the past three days, including weekend days [30]. The data from these dietary 
questionnaires were then analyzed using Nutrikcalß VO software (Consinfo, SC. Mexico) to identify the food groups ingested by the participants and estimate the total quantity of energy and nutrient consumption thereto.

\section{Measurements of adiponectin, insulin and inflammation markers}

The aliquoted serum was unfrozen to estimate serum adiponectin, insulin, interleukin 6 (IL-6), and C-reactive protein (CRP) levels using a sandwich enzyme-linked immunosorbent assay (ELISA) procedure. Total serum adiponectin and interleukin 6 (IL-6) were measured by the BioLegend ${ }^{\circledR}$ Human Acrp30 and IL-6 ELISA MAX Deluxe Kits (BioLegend CNS, Inc., CA, USA). Human Acrp30 sensitivity for this kit was reported by the manufacturer to be able to detect this molecule at concentrations as low as $31.3 \mathrm{pg} / \mathrm{mL}$, and at even lower amounts in some cases. The serum insulin levels were assessed by the DRG® human insulin ELISA kit (DRG Inc. NJ, USA); CRP serum levels were evaluated by the Cell Biolabs ${ }^{\circledR}$ ELISA Kit (Cell Biolabs, Inc. CA, USA). Standards and serum samples were plated in an antibody coated 96-well plate and processed according to the instructions of the ELISA kit providers. After the stop buffer solution was plated, absorbance was read at 450 and $570 \mathrm{~nm}$ on the plate reader Multiskan GO Microplate Spectrophotometer (Thermo Fisher Scientific ${ }^{\circledR}$, USA). To evaluate insulin resistance (IR), the homeostasis model assessment (HOMA-IR) was calculated using the HOMA index formula [fasting insulin $(\mu \mathrm{Ul} / \mathrm{mL}) \mathrm{x}$ fasting glucose $(\mathrm{mg} / \mathrm{dL}) / 405]$.

\section{Definitions and diagnostic criteria}

The cut-point criteria to consider IR status was a HOMA-IR index $\geq 2.5$ [31-33]; hypercholesterolemia was defined as total cholesterol $\geq 200 \mathrm{mg} / \mathrm{dL}$; hypertriglyceridemia was defined as triglycerides $\geq 150 \mathrm{mg} / \mathrm{dL}$; Low HDL-c was defined at levels $<40 \mathrm{mg} / \mathrm{dL}$ in men, and $<50 \mathrm{mg} / \mathrm{dL}$ in women [31]; the BMI categories were classified in underweight $\left(<18.5 \mathrm{Kg} / \mathrm{m}^{2}\right)$, normal weight $\left(18.5\right.$ to $\left.<25.0 \mathrm{Kg} / \mathrm{m}^{2}\right)$, overweight $\left(25\right.$ to $\left.<30 \mathrm{Kg} / \mathrm{m}^{2}\right)$; and obesity ( $\geq 30$ $\mathrm{Kg} / \mathrm{m}^{2}$ ) [29]; abdominal obesity was determined at a waist circumference $\geq 90$ centimeters (cm) in men, and $\geq$ $80 \mathrm{~cm}$ in women [31]; hypoadiponectinemia was defined as adiponectin levels $<3.0 \mu \mathrm{g} / \mathrm{mL}$ [32, 33]. Physical activity frequency was obtained through modification of the sedentary lifestyle classification test of Pérez-Rojas [34], subjects exercising > 90 minutes/week were considered active, whereas those with < 90 minutes/week were considered sedentary. Tobacco consumption was evaluated in those who had been passive or active smokers ( $>1$ time/week).

\section{Statistical analysis}

The data were analyzed using SPSS v20 software (SPSS Inc. Chicago, IL, USA). The descriptive characteristics of the sample were presented as mean and standard deviations. Frequency distributions were described in polychotomous and dichotomous categoric variables. Chi-square was used to test independent category comparisons. The Kolmogorov-Smirnov test was used to confirm normal distribution of continuous quantitative variables. Student's T test for independent samples, ANOVA one way, and Post-hoc tests were used to realize group comparisons. Simple correlation tests (Pearson and Kendalls tau_b) and a multiple regression model (ANCOVA) were performed to account for variation in serum adiponectin levels. Additionally, the Odds Ratio (OR) and 95\% confidence interval (IC) values were calculated to identify hypoadiponectinemia risk factors. A $P$-value of less than 0.05 was considered statistically significant. Calculation of sample size was done a priori based upon a formula to estimate population mean. Given a calculated standard deviation for adiponectin levels of $2.3 \mu \mathrm{g} / \mathrm{mL}$, a sample size of 81 participants was determined to be required to achieve a $0.5 \mu \mathrm{g} / \mathrm{mL}$ margin of error at a $95 \%$ confidence level [33]. Nonprobabilistic convenience sampling process was done to select the participants.

\section{Results}


Characteristics of Participants

A total number of 173 apparently healthy young subjects (80.3\% women), $19.3 \pm 1.32$ years old, were included in this study (Fig. 1). The analyzed sample was restricted to a young, university student population from the Los Altos, Jalisco region of Mexico. The locality distribution as to specific municipalities is shown in Fig. 2. Of these undergraduate students, $53.2 \%$ were studying nutrition, $28.3 \%$ were medical students, and $18.5 \%$ were nursing students. Notable differences were found between genders as to weight, waist circumference, body fat mass, triglycerides, HDL-c, VLDL-c and adiponectin levels $(p<0.05)$, as is shown in Table 1. 
Table 1

Comparison of general characteristics by male and female gender.

\begin{tabular}{|c|c|c|c|c|}
\hline Characteristics & $\begin{array}{l}\text { Total } \\
(n=173)\end{array}$ & $\begin{array}{l}\text { Male } \\
(n=34)\end{array}$ & $\begin{array}{l}\text { Female } \\
(n=139)\end{array}$ & $P$ \\
\hline Age (years) & $19.3 \pm 1.3$ & $19.3 \pm 1.4$ & $19.3 \pm 1.3$ & 0.827 \\
\hline Tobacco (\%) & $26.6 \%(n=46)$ & $35.3 \%(n=12)$ & $24.5 \%(n=34)$ & 0.218 \\
\hline Sedentary lifestyle (\%) & $60.5 \%(n=104)$ & $52.9 \%(n=18)$ & $62.6 \%(n=87)$ & 0.332 \\
\hline \multicolumn{5}{|c|}{ Anthropometric variables } \\
\hline Body weight (Kg) & $62.0 \pm 14.0$ & $73.7 \pm 16.3$ & $59.3 \pm 11.9$ & $<0.001^{*}$ \\
\hline $\mathrm{BMI}\left(\mathrm{Kg} / \mathrm{m}^{2}\right)$ & $22.9 \pm 4.1$ & $24.0 \pm 7.7$ & $22.5 \pm 3.9$ & 0.068 \\
\hline Waist (cm) & $74.0 \pm 10.9$ & $81.5 \pm 12.8$ & $72.2 \pm 9.6$ & $<0.001^{*}$ \\
\hline Body fat mass (\%) & $25.0 \pm 9.2$ & $17.8 \pm 9.0$ & $26.7 \pm 8.4$ & $<0.001^{*}$ \\
\hline \multicolumn{5}{|c|}{ Biochemical parameters and inflammatory markers } \\
\hline Glucose (mg/dL) & $89.9 \pm 6.9$ & $91.7 \pm 7.6$ & $89.5 \pm 6.7$ & 0.095 \\
\hline Triglycerides (mg/dL) & $80.19 \pm 41.2$ & $100.8 \pm 53.2$ & $75.2 \pm 36.2$ & $0.001 *$ \\
\hline Cholesterol (mg/DI & $157.9 \pm 31.1$ & $156.5 \pm 35.5$ & $158.3 \pm 30.1$ & 0.767 \\
\hline LDL-c (mg/dL) & $86.0 \pm 24.1$ & $88.2 \pm 29.5$ & $85.4 \pm 22.7$ & 0.557 \\
\hline VLDL-c (mg/dL) & $16.0 \pm 8.2$ & $20.2 \pm 10.6$ & $15.0 \pm 7.2$ & $0.001 *$ \\
\hline $\mathrm{HDL}-\mathrm{c}(\mathrm{mg} / \mathrm{dL})$ & $55.9 \pm 13.7$ & $48.2 \pm 11.5$ & $57.8 \pm 13.6$ & $<0.001^{*}$ \\
\hline Insulin $(\mu \mathrm{UI} / \mathrm{mL})$ & $13.0 \pm 8.4$ & $14.4 \pm 10.7$ & $12.6 \pm 7.7$ & 0.341 \\
\hline HOMA-IR & $2.8 \pm 1.9$ & $3.2 \pm 2.1$ & $2.7 \pm 1.8$ & 0.323 \\
\hline Adiponectin $(\mu \mathrm{g} / \mathrm{mL})$ & $3.9 \pm 2.2$ & $2.3 \pm 0.8$ & $4.3 \pm 2.6$ & $0.001 *$ \\
\hline IL-6 (pg/mL) & $2.4 \pm 2.6$ & $2.4 \pm 2.2$ & $2.3 \pm 2.7$ & 0.450 \\
\hline $\mathrm{CRP}(\mathrm{ng} / \mathrm{mL})$ & $0.3 \pm 0.5$ & $0.2 \pm 0.4$ & $0.4 \pm 0.5$ & 0.348 \\
\hline \multicolumn{5}{|l|}{ Dietetic characteristics } \\
\hline Energy (Kcal/day) & $1946.8 \pm 697.6$ & $2082.7 \pm 777.7$ & $1913.1 \pm 675.1$ & 0.205 \\
\hline Carbohydrates (\%) & $49.9 \pm 9.4$ & $49.7 \pm 6.2$ & $50.0 \pm 10.0$ & 0.855 \\
\hline Proteins (\%) & $16.6 \pm 4.1$ & $16.5 \pm 5.1$ & $16.7 \pm 3.8$ & 0.784 \\
\hline Lipids (\%) & $35.3 \pm 6.7$ & $35.0 \pm 6.3$ & $35.3 \pm 6.8$ & 0.814 \\
\hline Fiber (g) & $17.2 \pm 15.2$ & $15.2 \pm 8.0$ & $17.7 \pm 16.6$ & 0.783 \\
\hline
\end{tabular}

The data are shown as mean \pm standard deviation and percentages $\%(n)$ for categorical variables. Dietary intake assessment was done through 24-h and 3-day prospective food questionnaires which were analyzed using Nutrikcal ${ }^{\circledR}$ software. BMI: Body mass index; LDL-C: Low-density lipoprotein; VLDL-c: Very low-density lipoprotein; HDL-C: High-density lipoprotein; HOMA-IR: Homeostatic model assessment for insulin resistance; IL-6: Interleukin 6; CRP: C-reactive protein. * $p<0.01$. 


\begin{tabular}{|c|c|c|c|c|}
\hline Characteristics & $\begin{array}{l}\text { Total } \\
(n=173)\end{array}$ & $\begin{array}{l}\text { Male } \\
(n=34)\end{array}$ & $\begin{array}{l}\text { Female } \\
(n=139)\end{array}$ & $P$ \\
\hline Sodium (mg) & $2450.4 \pm 1612.3$ & $2642.8 \pm 1710.8$ & $2401.6 \pm 1589.2$ & 0.053 \\
\hline Cholesterol (mg) & $329.8 \pm 534.1$ & $285.6 \pm 153.7$ & $341.0 \pm 593.0$ & 0.372 \\
\hline \multicolumn{5}{|c|}{$\begin{array}{l}\text { The data are shown as mean } \pm \text { standard deviation and percentages } \%(n) \text { for categorical variables. Dietary intake } \\
\text { assessment was done through } 24-\text { h and } 3 \text {-day prospective food questionnaires which were analyzed using } \\
\text { Nutrikcal@ software. BMI: Body mass index; LDL-c: Low-density lipoprotein; VLDL-c: Very low-density lipoprotein } \\
\text { HDL-C: High-density lipoprotein; HOMA-IR: Homeostatic model assessment for insulin resistance; IL-6: Interleukir } \\
6 \text {; CRP: C-reactive protein. * } p<0.01 \text {. }\end{array}$} \\
\hline
\end{tabular}

In accordance with the WHO classification, the BMI categories found in this population were as follows: Underweight $10.4 \%$; Normal weight $66.5 \%$; Overweight/Pre-obesity $15.6 \%$, and Obesity $7.5 \%$. No significant differences in this classification were observed as between genders (data not shown). However, as is shown in Table 2, sedentarism and certain anthropometric and biochemical parameters including body weight, waist circumference, body fat mass, triglycerides, total cholesterol, VLDL-cholesterol, LDL-cholesterol, HDL-cholesterol, insulin, HOMA-IR, CRP and dietetic cholesterol consumption were statistically different as between the BMI categories $(p<0.01)$. 
Table 2

Comparison of general characteristics categorized by BMI.

\begin{tabular}{|c|c|c|c|c|c|}
\hline Characteristics & $\begin{array}{l}\text { Underweight } \\
(n=18)\end{array}$ & $\begin{array}{l}\text { Normal weight } \\
(n=115)\end{array}$ & $\begin{array}{l}\text { Overweight } \\
(n=27)\end{array}$ & $\begin{array}{l}\text { Obesity } \\
(n=13)\end{array}$ & $P$ \\
\hline Age (years) & $18.9 \pm 0.6$ & $19.5 \pm 1.6$ & $19.6 \pm 2.0$ & $18.9 \pm 2.0$ & 0.300 \\
\hline Tobacco (\%) & $27.8 \%(n=5)$ & $27.0 \%(n=31)$ & $25.9 \%(n=7)$ & $23.1 \%(n=3)$ & 0.989 \\
\hline Sedentarism (\%) & $94.4 \%(n=17)$ & $53.0 \%(n=61)$ & $74.1 \%(n=20)$ & $46.2 \%(n=6)$ & $<0.001^{\mathrm{a}}$ \\
\hline \multicolumn{6}{|c|}{ Anthropometric variables } \\
\hline Body weight (Kg) & $46.1 \pm 5.3$ & $58.0 \pm 7.3$ & $75.2 \pm 10.6$ & $93.0 \pm 9.1$ & $<0.001^{\mathrm{a}}$ \\
\hline Waist perimeter $(\mathrm{cm})$ & $61.9 \pm 3.6$ & $70.8 \pm 5.6$ & $84.3 \pm 7.3$ & $97.7 \pm 9.8$ & $<0.001^{\mathrm{a}}$ \\
\hline Body fat mass (\%) & $12.0 \pm 5.4$ & $23.5 \pm 6.6$ & $33.5 \pm 5.7$ & $39.4 \pm 4.8$ & $<0.001^{a}$ \\
\hline \multicolumn{6}{|c|}{ Biochemical parameters and inflammatory markers } \\
\hline Glucose (mg/dL) & $91.5 \pm 7.3$ & $89.7 \pm 5.9$ & $88.5 \pm 8.5$ & $92.3 \pm 10.2$ & 0.285 \\
\hline Triglycerides (mg/dL) & $64.9 \pm 19.2$ & $77.3 \pm 40.1$ & $88.1 \pm 49.5$ & $114.9 \pm 39.2$ & $0.004^{3}$ \\
\hline Cholesterol (mg/dL) & $144.0 \pm 29.3$ & $159.7 \pm 26.9$ & $152.0 \pm 27.0$ & $178.1 \pm 51.2$ & $0.014^{b}$ \\
\hline LDL-c (mg/dL) & $72.5 \pm 18.9$ & $85.7 \pm 20.0$ & $86.36 \pm 26.8$ & $110.6 \pm 39.1$ & $<0.001^{\text {bdef }}$ \\
\hline VLDL-c (mg/dL) & $13.0 \pm 3.8$ & $15.5 \pm 8.0$ & $17.6 \pm 9.9$ & $23.0 \pm 7.8$ & $0.004^{\mathrm{c}}$ \\
\hline HDL-c (mg/dL) & $58.6 \pm 15.1$ & $58.6 \pm 12.2$ & $48.0 \pm 13.4$ & $44.5 \pm 15.0$ & $<0.001^{\text {bdef }}$ \\
\hline Insulin ( $\mu \mathrm{UI} / \mathrm{mL})$ & $9.9 \pm 3.2$ & $11.4 \pm 5.3$ & $14.2 \pm 6.9$ & $27.7 \pm 17.8$ & $<0.001^{c}$ \\
\hline HOMA-IR & $2.3 \pm 0.8$ & $2.4 \pm 1.1$ & $3.2 \pm 1.6$ & $6.4 \pm 4.0$ & $<0.001^{\text {bdef }}$ \\
\hline Adiponectin $(\mu \mathrm{g} / \mathrm{mL})$ & $3.8 \pm 4.6$ & $4.2 \pm 2.3$ & $3.8 \pm 2.4$ & $3.2 \pm 2.2$ & 0.638 \\
\hline IL-6 (pg/mL) & $1.8 \pm 0.6$ & $2.2 \pm 2.5$ & $2.6 \pm 2.7$ & $3.5 \pm 3.9$ & 0.311 \\
\hline CRP (ng/mL) & $0.0 \pm 0.0$ & $0.2 \pm 0.4$ & $0.9 \pm 0.6$ & $0.4 \pm 0.6$ & $0.003^{\mathrm{de}}$ \\
\hline \multicolumn{6}{|l|}{ Dietetic characteristics } \\
\hline Energy (Kcal/day) & $2014.4 \pm 546$ & $1944.6 \pm 716$ & $1855.9 \pm 563$ & $2069.9 \pm 1001$ & 0.807 \\
\hline Carbohydrates (\%) & $48.1 \pm 4.9$ & $50.0 \pm 7.9$ & $51.3 \pm 15.3$ & $47.7 \pm 10.9$ & 0.578 \\
\hline Proteins (\%) & $16.4 \pm 3.2$ & $16.4 \pm 3.9$ & $17.6 \pm 4.4$ & $17.5 \pm 6.0$ & 0.529 \\
\hline \multicolumn{6}{|c|}{$\begin{array}{l}\text { The data are shown as mean } \pm \text { standard deviation and percentages } \%(\mathrm{n}) \text { for categorical variables. In accord } \\
\text { with the World Health Organization }(\mathrm{WHO}) \text {, the BMI }\left(\mathrm{Kg} / \mathrm{m}^{2}\right) \text { categories were classified as Underweight } \leq 18 \text {. } \\
\text { Normal weight }>18.5 \text { to }<25 ; \text { Overweight/Pre-obesity } \geq 25 \text { to }<29.9 \text {; Obesity } \geq 30) \text {. BMI: Body mass index; } \mathrm{L} \\
\text { Low-density lipoprotein; VLDL-c: Very low-density lipoprotein; HDL-C: High-density lipoprotein; HOMA-IR: } \\
\text { Homeostatic model assessment for insulin resistance; IL-6: Interleukin } 6 \text {; CRP: C-reactive protein. }{ }^{a} p<0.05 \\
\text { between all groups; }{ }^{\text {b }} p<0.01 \text { Underweight vs Obesity; }{ }^{\mathrm{C}} p<0.05 \text { Underweight } v s \text { Normal weight; }{ }^{\mathrm{d}} p<0.05 \\
\text { Underweight vs Overweight; }{ }^{\mathrm{e}} p<0.01 \text { Normal weight } v s \text { Overweight, }{ }^{\mathrm{f}} p<0.01 \text { Normal weight } v s \text { Obesity. }\end{array}$} \\
\hline
\end{tabular}




\begin{tabular}{|c|c|c|c|c|c|}
\hline Characteristics & $\begin{array}{l}\text { Underweight } \\
(n=18)\end{array}$ & $\begin{array}{l}\text { Normal weight } \\
(n=115)\end{array}$ & $\begin{array}{l}\text { Overweight } \\
(n=27)\end{array}$ & $\begin{array}{l}\text { Obesity } \\
(n=13)\end{array}$ & $P$ \\
\hline Lipids (\%) & $36.6 \pm 4.6$ & $35.4 \pm 7.0$ & $34.8 \pm 6.3$ & $35.4 \pm 6.7$ & 0.758 \\
\hline Fiber (g) & $15.1 \pm 7.2$ & $18.1 \pm 17.8$ & $15.3 \pm 9.2$ & $16.5 \pm 7.2$ & 0.764 \\
\hline Sodium (mg) & $2564 \pm 1259$ & $2399 \pm 1456$ & $2041 \pm 915$ & $3419 \pm 3404$ & 0.087 \\
\hline Cholesterol (mg) & $220.8 \pm 105.7$ & $303.4 \pm 291.1$ & $290.4 \pm 122.7$ & $786.2 \pm 1728.8$ & $0.0130^{\mathrm{b}}$ \\
\hline \multicolumn{6}{|c|}{ 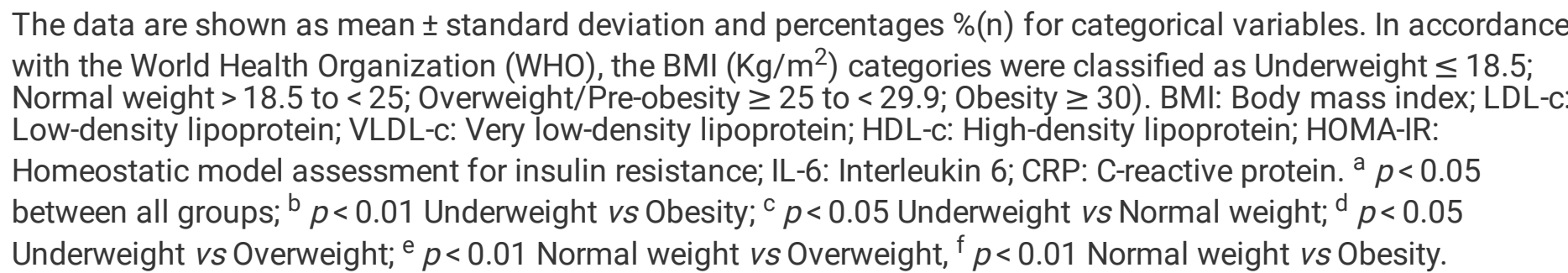 } \\
\hline
\end{tabular}

Adiponectin relationship to nutritional and metabolic traits

The total serum adiponectin level (all detected isoforms) was evaluated by ELISA technique resulting in an average serum adiponectin level of $3.96 \pm 2.21 \mu \mathrm{g} / \mathrm{mL}$ in the studied samples. In line with the study's purpose, serum adiponectin levels were classified in two categories: Normal adiponectin ( $\geq 3 \mu \mathrm{g} / \mathrm{mL}$ ) and hypoadiponectinemia (< $3.0 \mu \mathrm{g} / \mathrm{mL}$ ). This classification was implemented to identify any cross-sectional relationship between serum adiponectin levels and nutritional and/or biochemical characteristics. Review of results with respect to these classifications identified hypoadiponectinemia in $41.6 \%$ of the studied population. In addition, notable differences in anthropometric and biochemical variables including weight, waist circumference, LDL-c, HDL-c, VLDL-c, insulin and HOMA-IR were found as between these two adiponectin categories (Table 3). 
Table 3

Characteristics according to adiponectin categories.

\begin{tabular}{|c|c|c|c|}
\hline Characteristics & Normal adiponectin & Hypoadiponectinemia & $P$ \\
\hline \multicolumn{4}{|l|}{ Anthropometric variables } \\
\hline Weight (Kg) & $59.2 \pm 12.0$ & $66.9 \pm 14.6$ & $0.009 * \star$ \\
\hline $\mathrm{BMI}\left(\mathrm{Kg} / \mathrm{m}^{2}\right)$ & $22.5 \pm 3.9$ & $24.1 \pm 4.7$ & 0.079 \\
\hline Waist circumference $(\mathrm{cm})$ & $72.2 \pm 10.4$ & $78.4 \pm 10.0$ & $0.008^{\star \star}$ \\
\hline Body fat mass (\%) & $25.9 \pm 8.6$ & $25.4 \pm 11.2$ & 0.817 \\
\hline \multicolumn{4}{|c|}{ Biochemical and inflammatory markers } \\
\hline Glucose (mg/dL) & $89.7 \pm 6.6$ & $90.3 \pm 6.0$ & 0.693 \\
\hline Total cholesterol (mg/dL) & $150.2 \pm 36.5$ & $154.8 \pm 3.1$ & 0.467 \\
\hline $\mathrm{HDL}-\mathrm{c}(\mathrm{mg} / \mathrm{dL})$ & $60.6 \pm 14.0$ & $50.8 \pm 11.6$ & $0.001^{\star \star}$ \\
\hline LDL-c (mg/dL) & $86.1 \pm 25.7$ & $85.9 \pm 24.7$ & $0.033^{*}$ \\
\hline VLDL-c (mg/dL) & $13.5 \pm 5.9$ & $18.1 \pm 9.4$ & $0.006^{\star \star}$ \\
\hline Triglycerides (mg/dL) & $67.7 \pm 29.3$ & $90.6 \pm 46.9$ & 0.006 \\
\hline Insulin $(\mu \mathrm{UI} / \mathrm{mL})$ & $10.7 \pm 4.3$ & $15.9 \pm 12.0$ & $0.009 * \star$ \\
\hline HOMA-IR & $2.4 \pm 1.0$ & $3.6 \pm 28$ & $0.027 \star$ \\
\hline $\mathrm{IL}-6(\mathrm{pg} / \mathrm{mL})$ & $2.2 \pm 2.8$ & $2.7 \pm 3.2$ & 0.450 \\
\hline $\mathrm{CRP}(\mathrm{ng} / \mathrm{mL})$ & $0.2 \pm 0.4$ & $0.4 \pm 0.6$ & 0.348 \\
\hline
\end{tabular}

Considering diet as an environmental factor that relates to metabolic issues, the total dietary intake was evaluated through a 24-h and 3-day prospective dietetic questionnaires in order to identify any association between diet and serum adiponectin levels. The dietetic questionnaires were analyzed by registered nutritionists recruited for this study using Nutrikcal ${ }^{\circledR}$ software. This software provides a quantitative analysis of the total nutrient content from each dietetic questionnaire. After the dietary analyses were completed, dietary data including total 24-hour dietary energy intake, macronutrients (carbohydrates, proteins and lipids), micronutrients (vitamin and minerals), dietetic fiber and sugar were reported and averaged. In addition, examination was conducted regarding standard food servings consumed by participants (e.g., dairy products, legumes, vegetables, cereals, meat, fruits and oils), categorized by food groups as established in the Mexican Food Equivalent System, a dynamic meal-equivalent system that groups meals by their similar nutritional composition (energy and macronutrient content), as is shown in Fig. $3[35,36]$.

Findings in the studied sample revealed total caloric intake was in accordance with national dietary guidelines of the Mexican NOM-043-SSA2-2012 [35, 37]; whereas, the distribution of proteins and lipids (\%) was found higher than those reported in the national dietary guidelines. Average ingestion of carbohydrates was in line with the dietary 
guidelines; however, dietetic fiber, folic acid, vitamin E, and zinc intake were below those of the Mexican standard and likewise did not meet the Dietary References Intake (RDI) guidelines for the Mexican population [38].

Subsequently, the total nutrient consumption and quantity of standardized food servings were examined as between subjects who had normal adiponectin levels versus subjects who were found to have hypoadiponectinemia, the results shown in Table 4. Generally, no significant differences were found noting with exception that participants classified in the hypoadiponectinemia category consumed greater amounts of Vitamin B2 and calcium. It should also be noted that greater sodium consumption was found in those in the hypoadiponectinemia category versus those in the normal adiponectin category (3100.6 $\pm 2431.1 \mathrm{mg}$ and $2232.3 \pm 1165.8 \mathrm{mg}$, respectively, $p=0.053)$, as is shown in Table 4. 
Table 4

Dietetic analysis according to adiponectin categories.

\begin{tabular}{|c|c|c|c|}
\hline Variables & Normal adiponectin & Hypoadiponectinemia & $P$ \\
\hline Energy (Kcal/day) & $1991.6 \pm 176.7$ & $2054.9 \pm 197.4$ & 0.129 \\
\hline Carbohydrates (\%) & $51.1 \pm 13.2$ & $50.9 \pm 7.3$ & 0.939 \\
\hline Proteins (\%) & $16.9 \pm 3.7$ & $16.3 \pm 3.9$ & 0.441 \\
\hline Lipids (\%) & $35.6 \pm 7.7$ & $33.8 \pm 6.0$ & 0.241 \\
\hline Saturated fatty acids (\%) & $9.7 \pm 2.6$ & $9.6 \pm 2.8$ & 0.904 \\
\hline Monounsaturated fatty acids (\%) & $10.5 \pm 3.7$ & $10.5 \pm 3.5$ & 0.954 \\
\hline Polyunsaturated fatty acids (\%) & $4.5 \pm 1.7$ & $4.1 \pm 1.6$ & 0.210 \\
\hline Cholesterol (mg) & $318.0 \pm 403.3$ & $463.5 \pm 1045.5$ & 0.372 \\
\hline Fiber (g) & $17.8 \pm 11.5$ & $17.2 \pm 31.1$ & 0.783 \\
\hline \multicolumn{4}{|l|}{ Minerals } \\
\hline Sodium (mg) & $2232.3 \pm 1165.8$ & $3100.6 \pm 2431.1$ & 0.053 \\
\hline Cholesterol (mg) & $318.1 \pm 403.3$ & $463.4 \pm 1045.4$ & 0.373 \\
\hline Calcium (mg) & $837.2 \pm 373.1$ & $1064.4 \pm 593.8$ & $0.048 *$ \\
\hline Potassium (mg) & $1000.3 \pm 141.5$ & $1198.8 \pm 199.8$ & 0.784 \\
\hline Magnesium (mg) & $268.8 \pm 155.9$ & $241.7 \pm 105.3$ & 0.370 \\
\hline Phosphorus (mg) & $719.2 \pm 322.2$ & $915.3 \pm 683.8$ & 0.117 \\
\hline Selenium (mcg) & $36.4 \pm 30.8$ & $44.5 \pm 71.82$ & 0.476 \\
\hline Zinc (mg) & $7.3 \pm 3.1$ & $8.0 \pm 5.1$ & 0.441 \\
\hline \multicolumn{4}{|l|}{ Vitamins } \\
\hline Vitamin A (mcg) & $947.9 \pm 773.9$ & $1077.8 \pm 1024.4$ & 0.504 \\
\hline Vitamin B1 (mg) & $1.4 \pm 0.7$ & $1.5 \pm 0.9$ & 0.433 \\
\hline Vitamin B2 (mg) & $1.5 \pm 0.7$ & $2.2 \pm 1.8$ & $0.049 *$ \\
\hline Vitamin B3 (mg) & $16.9 \pm 7.4$ & $20.6 \pm 13.1$ & 0.118 \\
\hline Vitamin B5 (mg) & $2.9 \pm 2.5$ & $3.4 \pm 3.5$ & 0.412 \\
\hline Vitamin B6 (mg) & $1.5 \pm 0.7$ & $1.8 \pm 1.1$ & 0.208 \\
\hline Vitamin B9 (mcg) & $269.5 \pm 206.3$ & $309.4 \pm 224.8$ & 0.397 \\
\hline Vitamin B12 (mcg) & $3.9 \pm 4.4$ & $5.2 \pm 6.0$ & 0.276 \\
\hline Vitamin C (mg) & $113.5 \pm 178.1$ & $106.8 \pm 92.1$ & 0.836 \\
\hline Vitamin E (mg) & $2.3 \pm 1.9$ & $2.1 \pm 1.5$ & 0.725 \\
\hline
\end{tabular}


The data are shown as mean \pm standard deviation. Dietary intake assessment was done through 24-h and 3-day prospective food questionnaires which were analyzed using Nutrikcal ${ }^{\circledR}$ software. Hypoadiponectinemia was defined as serum adiponectin levels $<3.03 \mu \mathrm{g} / \mathrm{mL}[32,33]$. ${ }^{*} \mathrm{p}<0.05$.

Pearson and Kendall's tau_b correlations were then applied to the data to compare serum adiponectin levels to other established variables. Serum adiponectin levels correlated negatively to weight, waist circumference, abdominal obesity, Low-HDL-c, VLDL-c, triglycerides, insulin and HOMA-IR; whereas serum adiponectin levels positively correlated to the female gender, cholesterol and HDL-c, as shown in Table 5. 
Table 5

Correlations for adiponectin.

\begin{tabular}{|c|c|c|}
\hline Characteristics & Correlation coefficient & $P$ \\
\hline Female gender & 0.353 & $0.01 *$ \\
\hline Smoking & 0.047 & 0.597 \\
\hline Sedentarism & -0.028 & 0.755 \\
\hline \multicolumn{3}{|l|}{ Anthropometric variables } \\
\hline Weight & -0.238 & $0.014^{\star}$ \\
\hline BMI $\left(\mathrm{Kg} / \mathrm{m}^{2}\right)$ & -0.156 & 0.075 \\
\hline Waist circumference (cm) & -0.226 & $0.018^{*}$ \\
\hline Abdominal obesity & -0.196 & $0.028^{*}$ \\
\hline Body fat mass (\%) & 0.034 & 0.377 \\
\hline \multicolumn{3}{|c|}{ Biochemical and inflammatory markers } \\
\hline Glucose (mg/dL) & -0.134 & 0.107 \\
\hline Cholesterol (mg/dL) & 0.203 & $0.029 *$ \\
\hline $\mathrm{HDL}-\mathrm{c}(\mathrm{mg} / \mathrm{dL})$ & 0.487 & $0.01 *$ \\
\hline Low- HDL-c & -0.336 & $0.01 *$ \\
\hline LDL-c (mg/dL) & 0.066 & 0.271 \\
\hline VLDL-c (mg/dL) & -0.187 & $0.041^{\star}$ \\
\hline Triglycerides (mg/dL) & -0.187 & $0.041^{*}$ \\
\hline Hypertriglyceridemia & -0.162 & 0.065 \\
\hline Insulin ( $\mu \mathrm{UI} / \mathrm{mL})$ & -0.259 & $0.011^{*}$ \\
\hline HOMA-IR & -0.265 & $0.010^{*}$ \\
\hline $\mathrm{IL}-6(\mathrm{pg} / \mathrm{mL})$ & 0.145 & 0.090 \\
\hline $\mathrm{CRP}(\mathrm{mg} / \mathrm{L})$ & 0.012 & 0.473 \\
\hline \multicolumn{3}{|c|}{$\begin{array}{l}\text { Pearson and Kendall's tau_b correlations. Hypertriglyceridemia was defined as triglycerides } \geq 150 \mathrm{mg} / \mathrm{dL} \text {; low } \\
\text { HDL-c was considered at levels }<40 \mathrm{mg} / \mathrm{dL} \text { in men, and }<50 \mathrm{mg} / \mathrm{dL} \text { in women; abdominal obesity was } \\
\text { determined by waist circumference } \geq 90 \mathrm{~cm} \text { in men, and } \geq 80 \mathrm{~cm} \text { in women [33]; BMl: body mass index; LDL-c: } \\
\text { low-density lipoprotein; VLDL-c: very low-density lipoprotein; HDL-c: high-density lipoprotein; HOMA-IR: } \\
\text { homeostatic model assessment for insulin resistance; IL-6: interleukin 6; CRP: C-reactive protein. *p }<0.05 \text {. }\end{array}$} \\
\hline
\end{tabular}

In addition, Pearson and Kendall's tau_b correlation tests were used to investigate the relationship between serum adiponectin levels as compared with nutrient consumption and dietary patterns. A positive correlation was observed between adiponectin and fruit intake; whereas a negative correlation was observed between adiponectin and sodium intake. These correlations are shown in Table 6. 
Table 6

Correlations between adiponectin and dietetic characteristics.

\begin{tabular}{|c|c|c|}
\hline Nutrients & Correlation coefficient & $P$ \\
\hline Energy intake (Kcal/24 h) & -0.131 & 0.118 \\
\hline Protein (\%) & -0.044 & 0.345 \\
\hline Lipids (\%) & 0.044 & 0.343 \\
\hline Carbohydrates (\%) & 0.051 & 0.321 \\
\hline Cholesterol (mg) & -0.090 & 0.207 \\
\hline Sugar (g) & -0.140 & 0.102 \\
\hline Fiber (g) & -0.014 & 0.449 \\
\hline \multicolumn{3}{|l|}{ Vitamins } \\
\hline Vitamin A $(\mu \mathrm{g})$ & -0.001 & 0.496 \\
\hline Vitamin E (mg) & -0.002 & 0.493 \\
\hline Vitamin C (mg) & 0.142 & 0.099 \\
\hline Vitamin B1 (mg) & -0.080 & 0.234 \\
\hline Vitamin B2 (mg) & -0.165 & 0.067 \\
\hline Vitamin B3 (mg) & -0.102 & 0.177 \\
\hline Vitamin B5 (mg) & -0.103 & 0.175 \\
\hline Vitamin B6 (mg) & -0.126 & 0.126 \\
\hline Vitamin B12 $(\mu \mathrm{g})$ & -0.066 & 0.277 \\
\hline \multicolumn{3}{|l|}{ Minerals } \\
\hline Calcium (mg) & -0.134 & 0.113 \\
\hline Potassium (mg) & 0.034 & 0.381 \\
\hline Magnesium (mg) & 0.058 & 0.301 \\
\hline Sodium (mg) & -0.196 & $0.037 *$ \\
\hline \multicolumn{3}{|l|}{ Standard food servings } \\
\hline Dairy products & -0.014 & 0.450 \\
\hline Legumes & 0.53 & 0.316 \\
\hline Vegetables & 0.04 & 0.360 \\
\hline Cereals & -0.177 & 0.054 \\
\hline Meat & -0.108 & 0.163 \\
\hline Fruits & 0.204 & $0.031^{\star}$ \\
\hline Fat & -0.06 & 0.479 \\
\hline
\end{tabular}


Pearson and Kendall's tau_b correlations. Dietary intake assessment was done through 24-h and 3-day prospective food questionnaires which were analyzed using Nutrikcal® software. The number of standard food servings by food groups established by the Mexican Food Equivalent System were correlated with serum adiponectin levels by Pearson correlation test * $p<0.05$.

Multivariate Models

In order to define associations between the independent variables and adiponectin, a multivariate ANCOVA model was implemented taking into consideration all relevant variables and their corresponding statistical significance. The ANCOVA model revealed that $41.6 \%$ of the variation found in serum adiponectin levels is related to independent variables such as the female gender, glucose tertiles, low HDL-C, sodium and vitamin $C$ intake $\left(r^{2}=0.416, p<0.01\right)$, as shown in Table 7.

Table 7

Multivariate model for adiponectin.

\begin{tabular}{|c|c|c|c|c|}
\hline Independent variables & B coefficient & Beta coefficient & $95 \% \mathrm{Cl}$ & $P$ \\
\hline Female gender & 2.09 & 0.371 & 1.124 to 3.060 & $0.01^{\star}$ \\
\hline High glucose tertile & -0.637 & -0.234 & -1.102 to -0.172 & $0.01^{*}$ \\
\hline Low HDL-c & -2.169 & -0.443 & -3.021 to -1.318 & $0.01^{*}$ \\
\hline Sodium consumption (mg) & -0.000242 & -0.202 & -0.000447 to -0.000036 & $0.022^{*}$ \\
\hline Vitamin $\mathrm{C}$ consumption (mg) & 0.004 & 0.245 & 0.001 to 0.006 & $0.006^{*}$ \\
\hline
\end{tabular}

Then, to study hypoadiponectinemia risk factors, odds ratio (OR) values were calculated in subjects presenting low adiponectin levels (hypoadiponectinemia). In addition to the gender variable, metabolic and nutritional traits such as hypertriglyceridemia, low HDL-c, abdominal obesity, insulin resistance, hypercholesterolemia, and high LDL-c were selected to be examined given their statistical differences. As is shown in Fig. 4, statistical significance was found in two variables: female gender and low HDL-c levels. Female gender appeared to be a protective factor against hypoadiponectinemia $(\mathrm{OR}=0.063,95 \%$ IC $0.013-0.3, p<0.01)$; whereas participants with low HDL-c levels were found to have a greater risk for hypoadiponectinemia $(\mathrm{OR}=4.92,95 \%$ IC $1.8-13.3, \mathrm{p}<0.01)$.

\section{Discussion}

Mexico is among the most negatively affected countries by obesity-related comorbidities. Given the accelerated increase of chronic diseases worldwide, it is essential to take action to address this health problem [39]. In this focused study, the nutritional practices of a specific Mexican population were examined with an intent to elucidate a possible adiponectin association with nutritional and sociodemographic characteristics in young students from the Los Altos, Jalisco region of Mexico. Mexico is a pluricultural nation with diverse mestizo ethnic groups derived from a mixture of indigenous peoples of the Americas, Spanish-Europeans, and to lesser degree, those of African and Asian descent. Accordingly, a wide genetic diversity is found in the different regions of Mexico [40].

Looking at the region studied here, the Mexican state of Jalisco renders an important contribution to the Mexican economy, contributing approximately $10.8 \%$ of the gross domestic product (GDP) primarily through its agricultural and farming sectors. Jalisco's population is distributed in urban and rural municipalities, the latter of which including 
the Los Altos region, located primarily in the eastern part of the state of Jalisco [41]. Although approximately $60 \%$ of the population of Jalisco is concentrated in the capital city of Guadalajara, according to a 2015 report by the Institute of Statistical and Geographic Information for the state of Jalisco, some 399,724 inhabitants of the state, a relatively significant number, live in Los Altos Jalisco region [41, 42]. Given the Los Altos region is an important religious and cultural center where its inhabitants exhibit varying sociocultural characteristics and are known for preserving older traditions [7], this population was particularly an attractive research focus given their distinct nutritional and lifestyle practices rendering scientific and medical findings as to this population uniquely important to provide lasting benefits to this and other similarly-situated populations.

With respect to the BMI categories, a considerable amount of malnutrition was discovered (33.5\%), distributed across underweight $(10.4 \%)$, overweight (15.6\%), and obesity (7.5\%) categories. It should be noted the results of this study contrasted with those reported in the National ENSANUT-2018 Survey with respect to obesity. The ENSANUT-2018 Survey reported obesity in $36.1 \%$ of adult subjects ( $>20$ years old) and in $14.6 \%$ of adolescents (12-19 years old), both results some two times greater or more those that found in this study $(7.5 \%)$ for the young student population. The overweight prevalence was comparable; the underweight prevalence could not be compared as it was not undertaken in the ENSANUT-2018 Survey. The ENSANUT-2016 Survey reported an underweight prevalence of $1.3 \%$ in both female and male adults from the central region of Mexico, the same general area where the Los Altos region is located. Interestingly, this study found an overall underweight prevalence of $7.7 \%$ with respect to females and $10.4 \%$ with respect to females and males combined [43], some six to ten times greater as those reported in the ENSANUT2016 Survey. This discrepancy may be due to certain societal influences on women to maintain a specific weight as it relates to body image (auto-perception), particularly given these women living in rural areas may possess fewer opportunities for professional development given they would need to migrate to another city to pursue university studies or a career, and as such, have more available time for self-evaluation in regards to their physical appearance [44]. Furthermore, cultural influences in rural regions of Mexico militate towards women being homemakers and mothers caring for babies and away from women pursuing other activities, perhaps also contributing to this discrepancy. While not a principal focus of this study, the issue of education and professional development of women living in rural areas remains an important topic to be discussed and studied, particularly in Mexico.

A notable difference was found in serum adiponectin levels by gender with women showing higher adiponectin levels in comparison to men $(4.3 \pm 2.6 \mu \mathrm{g} / \mathrm{mL}$ and $2.3 \pm 0.8 \mu \mathrm{g} / \mathrm{mL}$, respectively, $\mathrm{p}=0.001)$. Indeed, the female gender was identified as a protective factor against hypoadiponectinemia $(\mathrm{OR}=0.063,95 \% \mathrm{Cl} 0.013-0.3, \mathrm{p}<0.01)$. This gender disparity in adiponectin levels has been previously reported by Nishizawa and colleagues (2002). The Nishizawa study demonstrated that plasma adiponectin levels were 35\% lower in Japanese adult men (aged $52.6 \pm 11.9$ years) as compared to Japanese adult women (aged $53.2 \pm 12.0$ years). No significant differences in serum glucose or insulin levels were found by gender in that study. Additionally, it has been shown that in ICR ovariectomized female mice plasma adiponectin was not altered by the procedure; conversely, elevated levels of plasmatic adiponectin were detected in ICR castrated male mice. That study concluded that the presence of testosterone reduced plasma adiponectin levels, this finding confirmed by a cell culture of 3T3-L1 adipocytes exposed to testosterone with resulting decrease in adiponectin concentration in the culture media. That study also suggested the gender influence on adiponectin may be related to the higher risk of insulin resistance and atherosclerosis in males, as induced by androgens [45-47]. However, the physiological mechanism to explain this inverse relationship between adiponectin [mostly of the higher molecular weight (HMW) adiponectin isoform] and testosterone as between women and men is not yet clear [48,49]. Interestingly, the study by Leffler and colleagues (2019) shows that altered expression of the estrogen receptor $(\mathrm{ERa})$ in mice leads to interruption of adiponectin signaling, suggesting that adiponectin may have a physiological role association with hormonal ERa [50]. Also noteworthy is the previous research of Andersen (2007) 
and Böttner (2004) that reported a significant decrease of adiponectin levels associated with increased androgen levels found in boys who were initiating puberty $[46,51]$. Hence, several important studies attribute the adiponectin discrepancy between genders as being related to sex hormone influences [46, 52].

Interestingly, in this study, adiponectin levels were positively correlated with HDL-c (Pearson's $r=0.487, p=0.01$ ). Increased risk of hypoadiponectinemia was observed in subjects who exhibited low HDL-c levels $(O \mathrm{R}=4.92,95 \% \mathrm{Cl}$ $1.8-13.3, p<0.01)$. These findings coincide with previous studies suggesting a positive correlation between adiponectin and the HDL-c lipoprotein. In this context, Wang (2017) investigated the adiponectin role in macrophage reverse cholesterol transport through an in vivo study realized in adiponectin -/-knockout mice treated with intraperitoneal injections of different adiponectin concentrations. After four weeks, serum lipidic profiles were compared to the degree of severity of atherosclerotic lesions of the aorta by histopathological examination, as compared to a control group that received only saline injections (placebo). Interestingly, a significant reduction of the evaluated parameters was shown, including triglycerides, total cholesterol and LDL-c in the test group. Notably, HDLc levels were significantly increased. In addition, the degree of severity of atherosclerotic damage was reduced in the adiponectin-treated mice as compared to the control group. Also evaluated was the mRNA expression of the $A B C A 1$ gene by real time polymerase chain reaction (RT-PCR) along with the protein levels by Western blot in hepatic tissue. There, the ABCA1 protein and mRNA expression increased significantly in mice treated with adiponectin and the increase was found to be directly proportional to adiponectin concentration. Hence, this suggests that adiponectin plays a key role in the lipoprotein metabolism through the biosynthesis of HDL-c by modulation of the ABCA1 protein $[53,54]$. Furthermore, Tsubakio-Yamamoto and colleagues (2012) reported a positive correlation between adiponectin and HDL-c; whereas a negative correlation was observed between adiponectin and specific cholesterol types, particularly the larger sized VLDL molecules and the smaller, dense LDL molecules. Thus, the TsubakioYamamoto study suggested that variance in adiponectin levels may be related to the molecular size of the LDL molecule and the synthesis of HDL molecules (Tsubakio-Yamamoto, 2012). In the present study, a significant difference in HDL-c levels was detected between female and male participants wherein females had higher HDL-c levels than males [55]. It was not possible to discard or confirm a possible interaction between HDL-c and the female gender in regulating adiponectin levels in this study; however, this potential interaction would provide a most interesting opportunity for a follow-up study in the future.

Additionally, this study evaluated dietetic values to determine if a relationship existed between dietary characteristics and adiponectin levels in the target population. Habitual ingestion patterns of the studied population along with corresponding nutrients and caloric intake were compared to national and international guidelines. An adequate energy intake was found in this population overall; whereas, the consumption of fat, proteins and some vitamins including folic acid, vitamin B5 and minerals such as sodium, zinc, phosphorus and selenium, were not consumed in sufficient amounts per RDI standards (data not shown) [38,56,57]. The potential association between adiponectin levels and the quantity and quality of nutrients ingested was examined and a positive correlation was discovered as between adiponectin levels and fruit consumption. To a lesser degree, a positive correlation was also discovered between adiponectin levels and vitamin $\mathrm{C}$ ingestion. Finally, a negative correlation was discovered between adiponectin levels and sodium consumption pursuant to the ANCOVA multivariate model utilized.

In this respect, Corbi and colleagues (2019) similarly researched changes in adiponectin levels as compared to certain biochemical and anthropometric variables in severely obese persons after a nutritional intervention, including a fiber-rich, hypocaloric diet $(1200 \mathrm{Kcal} / \mathrm{d})$, and a four-week physical exercise program was administered. At the conclusion of the intervention, all participants there showed notable reduction in BMI scores and a significant increase in adiponectin levels. Additionally, the effect of genetic variants of the ADIPOQ gene was analyzed (the gene 
that encodes adiponectin), identifying the most accurate predictor of adiponectin variance as the wild allele for the single nucleotide polymorphism (SNP) c. $268 \mathrm{G}>\mathrm{A}$ in the ADIPOQ gene [58]. The present study does not attribute adiponectin level modulation to a single nutrient given the large variation of diet, food and beverage consumption encountered in the studied population in addition to the lack of a specific intervention being implemented in this study. For these reasons, further research is required to establish a more refined correlation as between adiponectin levels and specific dietary components, such as those found in the traditional Mexican diet. Thus, a future study could incorporate a planned nutritional intervention based on the traditional Mexican diet to reach further conclusions with respect to studying the potential effect of typical Mexican diet on adiponectin levels.

\section{Conclusions}

In summary, in this population a prevalence of underweight participants was identified, particularly with respect to young females. In addition, adiponectin level was positively associated with the female gender, HDL-c serum levels, and to a lesser degree, with certain dietary factors such as fruit, and vitamin C intake. A negative correlation was found between adiponectin level and sodium intake. This study, as with other cross-sectional research, had limitations in establishing the causality of changes to adiponectin levels. In this regard, and to better understand the effect of nutritional traits on serum adiponectin levels (particularly when using adiponectin levels as early chronic disease biomarkers), further research is necessary. Nonetheless, sufficient evidence exists to support nutritional interventions to diminish and/or prevent obesity-related diseases that, ideally, could reduce the negative impact of obesity in this studied population. External validity of the findings made herein remains an important study question that should be addressed in further research given the distinct nutritional and lifestyle practices of the Los Altos, Jalisco region target population, such studies possibly rendering important scientific and medical findings that will provide lasting benefits to not only this, but also to other similarly-situated populations.

\section{Abbreviations}

BMI: Body mass index; HDL-c:High-density lipoprotein cholesterol; VLDL-c:Very-low density cholesterol; LDL-c:Lowdensity Lipoprotein OECD:Organization for Economic Cooperation and Development; WHO:World Health Organization; ENSANUT:National Health and Nutrition Inquest; NCP:Nutrition Care Process; ISAK:International Society for the Advancement of Kinanthropometry; Acrp30:30 kDa adipocyte complement-related protein; Gbp28:28 kDa gelatin-binding protein; NF-кB:nuclear factor кB; IL-6:interleukin 6, CRP:C-reactive protein; IR:Insulin resistance; ELISA:sandwich enzyme-linked immunosorbent assay; HOMA-IR:Homeostasis model assessment for insulin resistance; RDI:Dietary References Intake.

\section{Declarations}

\section{Availability of data and materials}

The data bases from this research are available from the corresponding author on reasonable request.

\section{Consent for publication}

Not applicable.

\section{Ethics approval and consent to participate}


This research project was approved and registered by the Research and Bioethics Committee of the Los Altos University Center, Mexico, [CUA/CEI/027/2019]. All the student volunteers participating in the study provided voluntarily signed, written consent forms.

\section{Competing interests}

The authors declare no conflicts of interest.

\section{Funding}

This research received financial funding by PRODEP through grant 2017 CA-UDG-899 "Fortalecimiento de Cuerpos Academicos" and by CONACyT, Fondo Sectorial para la Educación, grant number CB-2015-254174.

\section{Author's Contributions}

“Conceptualization, M.R.G.G., Y.S.G.T.; methodology, Y.S.G.T., N.G.S., Z.V.d.I.M, L.F.G.R., C.M.R.R. and A.P.R.; formal analysis, M.R.G.G. and Y.S.G.T.; investigation, Y.S.G.T., P.N.V.B, S.S.E. and M.R.G.G.; resources, Y.S.G.T., M.R.G.G., N.G.S., Z.V.d.I.M., T.G.I., E.A.R.L., P.N.V.B, S.S.E.; writing-original draft preparation, M.R.G.G. and Y.S.G.T.; writingreview and editing S.S.E.; supervision, P.N.V.B., S.S.E.; project administration, M.R.G.G.; funding acquisition, M.R.G.G. All authors have read and agreed to the published version of the manuscript."

\section{Acknowledgments}

All the authors gratefully give thanks to Dr. Dennis S. Morris, Professor of Medicine UAG, for his review of this article and English language support. Also, the authors wish to thank Edgar Uriel Angel Ascencio, Patricia Martín del Campo Rayas, Alma Delia Ibarra Ramírez, Brenda Giselle Martínez Casarrubias, Wendy Saraí Jaramillo Gómez and Iván Arias Sanchéz for their technical support.

\section{References}

1. Schetz M, De Jong A, Deane AM, Druml W, Hemelaar P, Pelosi P, et al. Obesity in the critically ill: a narrative review. Intensive Care Med. 2019;45:757-69.

2. Poobalan A, Aucott L. Obesity Among Young Adults in Developing Countries: A Systematic Overview. Curr Obes Rep. 2016;5:2-13.

3. Obesity-Update-2017. https://www.oecd.org/health/health-systems/Obesity-Update-2017.pdf Internet. Accessed 17 may 2020.

4. Ensanut_2018_presentacion_resultados.

https://ensanut.insp.mx/encuestas/ensanut2018/doctos/informes/ensanut_2018_presentacion_resultados.pdf. Accessed 17 may 2020.

5. Rtveladze K, Marsh T, Barquera S, Sanchez Romero LM, Levy D, Melendez G, et al. Obesity prevalence in Mexico: impact on health and economic burden. Public Health Nutr. 2014;17:233-9.

6. Latnovic L, Rodriguez Cabrera L. Public health strategy against overweight and obesity in Mexico's National Agreement for Nutritional Health. Int J Obes Suppl. 2013;3:12-4. 
7. Martínez R. Regional Development Through Religious Tourism. San Juan de los Lagos and Jalostotitlán in Los Altos de Jalisco Region, Mexico. Études Caribéennes. http://journals.openedition.org/etudescaribeennes/5352. Accessed 20 Apr 2020.

8. Barrera-Cruz A, Rodríguez-González A, Molina-Ayala MA. Escenario actual de la obesidad en México. Rev Med Inst Mex Seguro Soc. 2013;3:292-99.

9. de Castro Ferreira Vicente SE, Corgosinho FC, da Silveira Campos RM, Landi Masquio DC, Oliveira e Silva L, Pelissari Kravchychyn AC, et al. The impact of adiponectin levels on biomarkers of inflammation among adolescents with obesity. Obes Med. 2017;5:4-10.

10. Gasparotto AS, Borges DO, Zandoná MR, Ramos MJ, Meihnardt NG, Mattevi VS. Adiponectin promoter polymorphisms are predictors of lipid profile improvement after bariatric surgery. Genet Mol Biol. 2017;40:73642.

11. Sparrenberger K, Sbaraini M, Cureau FV, Teló GH, Bahia L, Schaan BD. Higher adiponectin concentrations are associated with reduced metabolic syndrome risk independently of weight status in Brazilian adolescents. Diabetol Metab Syndr. 2019;11:40.

12. Hafiane A, Gasbarrino K, Daskalopoulou SS. The role of adiponectin in cholesterol efflux and HDL biogenesis and metabolism. Metabolism. 2019;100:153953.

13. Achari AE, Jain SK. Adiponectin, a Therapeutic Target for Obesity, Diabetes, and Endothelial Dysfunction. Int J Mol Sci. 2017;18:1321.

14. Fasshauer M, Blüher M. Adipokines in health and disease. Trends Pharmacol Sci. 2015;36:461-70.

15. Ouchi N, Parker JL, Lugus JJ, Walsh K. Adipokines in inflammation and metabolic disease. Nat Rev Immunol. 2011;11:85-97.

16. Ruan H, Dong LQ. Adiponectin signaling and function in insulin target tissues. J Mol Cell Biol. 2016;8:101-9.

17. Sciarretta S, Frati G. The Importance of Restoring the Adiponectin Signaling Pathway to Reduce Myocardial Reperfusion Injury in Diabetes. Diabetes. 2016;65:826-8.

18. Frankenberg AD von, Reis AF, Gerchman F. Relationships between adiponectin levels, the metabolic syndrome, and type 2 diabetes: a literature review. Arch Endocrinol Metab. 2017;61:614-22.

19. Pinheiro Volp AC. Los nutrientes pueden modular las concentraciones de adiponectina en adultos jóvenes aparentemente sanos. Nutr Hosp. 2016;33:580-7.

20. Silva FM, de Almeida JC, Feoli AM. Effect of diet on adiponectin levels in blood. Nutr Rev. 2011;69:599-612.

21. Attar MJH, Mohammadi S, Karimi M, Hosseinnezhad A, Hosseini SH, Eshraghian MR, et al. Association of adiponectin with dietary factors and cardiovascular risk factors in type 2 diabetes mellitus patients. Diabetes Metab Syndr Clin Res Rev. 2013;7:3-7.

22. Izadi V, Azadbakht L. Specific dietary patterns and concentrations of adiponectin. J Res Med Sci Off J Isfahan Univ Med Sci. 2015;20:178-84.

23. Spadafranca A, Piuri G, Bulfoni C, Liguori I, Battezzati A, Bertoli S, et al. Adherence to the Mediterranean Diet and Serum Adiponectin Levels in Pregnancy: Results from a Cohort Study in Normal Weight Caucasian Women. Nutrients. 2018;10:928.

24. Valerino-Perea. Lara-Castor, Armstrong, Papadaki. Definition of the Traditional Mexican Diet and Its Role in Health: A Systematic Review. Nutrients. 2019;11:2803.

25. Swan WI, Pertel DG, Hotson B, Lloyd L, Orrevall Y, Trostler N, et al. Nutrition Care Process (NCP) Update Part 2: Developing and Using the NCP Terminology to Demonstrate Efficacy of Nutrition Care and Related Outcomes. J

Page 22/32 
Acad Nutr Diet. 2019;119:840-55.

26. Stewart A, Marfell-Jones M. International Society for Advancement of Kinanthropometry. International standards for anthropometric assessment. Lower Hutt: International Society for the Advancement of Kinanthropometry; 2011.

27. Body Mass Index (BMI). https://www.who.int/data/gho/data/themes/theme-details/GHO/body-mass-index(bmi). Accessed 17 may 2020.

28. Knopfholz J, Disserol CCD, Pierin AJ, Schirr FL, Streisky L, Takito LL, et al. Validation of the Friedewald Formula in Patients with Metabolic Syndrome. Cholesterol. 2014:261878.

29. Castell GS, Serra L, Ribas-Barba L. ¿Qué y cuánto comemos? El método Recuerdo de 24 horas. Rev Esp Nutr Comunitaria. 2015;2:42-4.

30. Ortega R, Perez-Rodrigo C, López-Sobaler AM. Métodos de evaluación de la ingesta actual: registro o diario diétetico. Rev Esp Nutr Comunitaria. 2015;2:34-41.

31. González-Chávez A. Prevalencia del síndrome metabólico entre adultos mexicanos no diabéticos, usando las definiciones de la OMS, NCEP-ATPIIla e IDF. Rev Med Hosp Gen Mex. 2008;71:11-9.

32. Martinez-Lopez E, Garcia-Garcia MR, Gonzalez-Avalos JM, Maldonado-Gonzalez M, Ruiz-Madrigal B, Vizmanos $B$, et al. Effect of Ala54Thr polymorphism of FABP2 on anthropometric and biochemical variables in response to a moderate-fat diet. Nutr. 2013;29:46-51.

33. Garcia-Garcia MR, Morales-Lanuza MA, Campos-Perez WY, Ruiz-Madrigal B, Maldonado-Gonzalez M, Vizmanos B, et al. Effect of the ADIPOQ Gene - 11391G/A Polymorphism Is Modulated by Lifestyle Factors in Mexican Subjects. J Nutr Nutr. 2014;7:210-22.

34. García-Puello F, Herazo-Beltrán Y, Vidarte-Claros JA, García-Jimenez R, Crissien-Quiroz E. Evaluación de los niveles de actividad física en universitarios mediante método directo. Rev Salud Pública. 2018;20:606-11.

35. Perez-Lizaur AB, Gonzalez-Palacios B, Casstro-Becerra AL. Sistema Mexicano de Alimentos Equivalentes. Formatos de Nutricion y Salud, 2008.

36. Limon-Miro AT, Lopez-Teros V, Astiazaran-Garcia H. Dynamic Macronutrient Meal-Equivalent Menu Method: Towards Individual Nutrition Intervention Programs. Methods Protoc. 2019;2:78.

37. FAO.org:NOM-043-SSA2-2012 http://www.fao.org/faolex/results/details/en/c/LEX-FAOC119760/. Accessed 20 jun 2019.

38. FAO.org:NOM-247-SSA1-2008. http://www.fao.org/faolex/results/details/es/c/LEX-FAOC088981/. Accessed 20 jun 2019.

39. DiBonaventura MD, Meincke H, Le Lay A, Fournier J, Bakker E, Ehrenreich A. Obesity in Mexico: prevalence, comorbidities, associations with patient outcomes, and treatment experiences. Diabetes Metab Syndr Obes Targets Ther. 2017;11:1-10.

40. Velázquez A, Bilbao G, González-Trujillo JL, Hernández D, Pérez-Andrade ME, Vela M, et al. Apparent higher frequency of phenylketonuria in the Mexican state of Jalisco. Hum Genet. 1996;97:99-102.

41. Mariscal M, Salazar RM. Policentrismo y jerarquía de localidades de la región “Altos Sur" de Jalisco, 2010. ACE Archit City Environ. 2018;(38):235-62.

42. Altos-Sur-Diagnóstico. https://iieg.gob.mx/ns/wp-content/uploads/2019/06/03-Altos-Sur-Diagn\%C3\%B3stico. Accessed 17 may 2020.

43. Salud S de. Encuesta Nacional de Salud y Nutrición de Medio Camino 2016 http://www.gob.mx/salud/documentos/encuesta-nacional-de-salud-y-nutricion-de-medio-camino-2016.

Page 23/32 
Accessed 17 may 2020.

44. Paredes KF, Reséndiz FJD, Bautista-Día ML. Estatus de peso, conductas alimentarias de riesgo e insatisfacción corporal en mujeres adolescentes y jóvenes. Arch Med Col. 2019;19:303-12.

45. Nishizawa H, Shimomura I, Kishida K, Maeda N, Kuriyama H, Nagaretani H, et al. Androgens Decrease Plasma Adiponectin, an Insulin-Sensitizing Adipocyte-Derived Protein. Diabetes. 2002;51:2734-41.

46. Andersen KK, Frystyk J, Wolthers OD, Heuck C, Flyvbjerg A. Gender Differences of Oligomers and Total Adiponectin during Puberty: A Cross-Sectional Study of 859 Danish School Children. J Clin Endocrinol Metab. 2007;92:1857-62.

47. Dutta S, Sengupta P, Biswas A. Adiponectin in male reproduction and infertility. Asian Pac J Reprod. 2019;8:244.

48. Tilg $\mathrm{H}$, Moschen AR. Adipocytokines: mediators linking adipose tissue, inflammation and immunity. Nat Rev Immunol. 2006;6:772-83.

49. Snijder MB, Heine RJ, Seidell JC, Bouter LM, Stehouwer CDA, Nijpels G, et al. Associations of Adiponectin Levels With Incident Impaired Glucose Metabolism and Type 2 Diabetes in Older Men and Women: The Hoorn Study. Diabetes Care. 2006;29:2498-503.

50. Leffler KE, Abdel-Rahman AA. Estrogen-Dependent Disruption of Adiponectin-Connexin43 Signaling Underlies Exacerbated Myocardial Dysfunction in Diabetic Female Rats. J Pharmacol Exp Ther. 2019;368:208-17.

51. Böttner A, Kratzsch J, Müller G, Kapellen TM, Blüher S, Keller E, et al. Gender Differences of Adiponectin Levels Develop during the Progression of Puberty and Are Related to Serum Androgen Levels. J Clin Endocrinol Metab. 2004;89:4053-61.

52. Isobe T, Saitoh S, Takagi S, Takeuchi H, Chiba Y, Katoh N, et al. Influence of gender, age and renal function on plasma adiponectin level: the Tanno and Sobetsu study. Eur J Endocrinol. 2005;153:91-8.

53. Wang Y, Wang X, Guo Y, Bian Y, Bai R, Liang B, et al. Effect of adiponectin on macrophage reverse cholesterol transport in adiponectin-/-mice and its mechanism. Exp Ther Med. 2017;13:2757-62.

54. Marsche G, Zelzer S, Meinitzer A, Kern S, MeissI S, Pregartner G, et al. Adiponectin Predicts High-Density Lipoprotein Cholesterol Efflux Capacity in Adults Irrespective of Body Mass Index and Fat Distribution. J Clin Endocrinol Metab. 2017;102:4117-23.

55. Tsubakio-Yamamoto K, Sugimoto T, Nishida M, Okano R, Monden Y, Kitazume-Taneike R, et al. Serum adiponectin level is correlated with the size of HDL and LDL particles determined by high performance liquid chromatography. Metab - Clin Exp. 2012;61:1763-70.

56. Hernández Triana M. Recomendaciones nutricionales para el ser humano: actualización. Rev Cuba Investig Bioméd. 2004;23:266-92.

57. García Gabarra A. Ingesta de nutrientes: conceptos y recomendaciones internacionales. Nutr Hosp. 2006;21:291-9.

58. Corbi G, Polito R, Monaco ML, Cacciatore F, Scioli M, Ferrara N, et al. Adiponectin Expression and Genotypes in Italian People with Severe Obesity Undergone a Hypocaloric Diet and Physical Exercise Program. Nutrients. 2019;11:2195.

\section{Figures}




\begin{tabular}{|c|c|c|c|c|c|c|}
\hline \multirow{2}{*}{$\begin{array}{c}\text { Food } \\
\text { Groups }\end{array}$} & \multirow[b]{2}{*}{ Food Items } & \multirow{2}{*}{$\begin{array}{c}\text { Standard food } \\
\text { servings }\end{array}$} & \multicolumn{4}{|c|}{ Nutritional composition } \\
\hline & & & $\begin{array}{c}\text { Energy } \\
\text { (Kcal) }\end{array}$ & $\begin{array}{l}\begin{array}{l}\text { Protein } \\
\text { (grams) }\end{array} \\
\end{array}$ & $\begin{array}{c}\text { Fat } \\
\text { (grams) }\end{array}$ & $\begin{array}{c}\begin{array}{c}\text { Carbohydrates } \\
\text { (grams) }\end{array} \\
\end{array}$ \\
\hline \multirow[t]{3}{*}{ Vegetables } & $\begin{array}{l}\text { Spinach, chard, mushrooms, } \\
\text { lettuce, cucumber, radish (raw) }\end{array}$ & 2 cups & & & & \\
\hline & Nopal, squash, pumpkin,tomato & 1 cup & 25 & 2 & 0 & 4 \\
\hline & $\begin{array}{l}\text { Swiss chard, cauliflower, pea, } \\
\text { broccoli, carrot }\end{array}$ & $1 / 2$ cup & & & & \\
\hline \multirow[t]{5}{*}{ Fruits } & Grapes, tejocotes & 10 pieces & & & & \\
\hline & Peach, prickly pear, (small) & 2 pieces & & & & \\
\hline & $\begin{array}{l}\text { Apple, pomegranate, orange } \\
\text { and passion fruit, (small) }\end{array}$ & 1 piece & 60 & 0 & 0 & 15 \\
\hline & Banana, pear, grapefruit & $1 / 2$ piece & & & & \\
\hline & $\begin{array}{l}\text { Pineapple, watermelon, } \\
\text { cantaloupe, strawberry, papaya }\end{array}$ & 1 cup & & & & \\
\hline \multirow[t]{4}{*}{ Cereals } & Corn or wheat tortilla (small) & 1 piece & & & & \\
\hline & $\begin{array}{l}\text { Rice, corn kernels, pasta } \\
\text { (cooked), whole grain flakes }\end{array}$ & $1 / 2$ cup & & & & \\
\hline & Whole meal rye bread or toast & 1 slice & 70 & 2 & 0 & 15 \\
\hline & Corn cob & $3 / 4$ piece & & & & \\
\hline Legumes & Bean, lentil, chickpea & $1 / 2$ cup & 120 & 8 & 1 & 20 \\
\hline \multirow{3}{*}{$\begin{array}{l}\text { Food of } \\
\text { animal } \\
\text { source }\end{array}$} & $\begin{array}{l}\text { Beef steak, chicken or turkey } \\
\text { breast, tuna }\end{array}$ & 30 grams & & & & \\
\hline & & & 75 & 7 & 5 & 0 \\
\hline & Whole egg & 1 piece & & & & \\
\hline \multirow[t]{3}{*}{$\begin{array}{l}\text { Dairy } \\
\text { products }\end{array}$} & Milk, jocoque & 1 cup & & & & \\
\hline & & & 150 & 9 & 8 & 12 \\
\hline & Natural yogurt & $3 / 4$ cup & & & & \\
\hline \multirow[t]{3}{*}{ Oils } & Avocado & $1 / 4$ piece & & & & \\
\hline & & & 45 & 0 & 5 & 0 \\
\hline & $\begin{array}{l}\text { Peanuts, sunflower and seeds } \\
\text { oils }\end{array}$ & 1 tablespoon & & & & \\
\hline
\end{tabular}

\section{Figure 1}

Numbers of individuals at each stage of study. Participants' eligibility and non-inclusion are represented according to the Los Altos University Center target population. 


\begin{tabular}{|c|c|c|c|c|c|c|}
\hline \multirow{2}{*}{$\begin{array}{l}\text { Food } \\
\text { Groups }\end{array}$} & \multirow[b]{2}{*}{ Food Items } & \multirow{2}{*}{$\begin{array}{l}\text { Standard food } \\
\text { servings }\end{array}$} & \multicolumn{4}{|c|}{ Nutritional composition } \\
\hline & & & $\begin{array}{c}\begin{array}{c}\text { Energy } \\
\text { (Kcal) }\end{array} \\
\end{array}$ & $\begin{array}{l}\begin{array}{l}\text { Protein } \\
\text { (grams) }\end{array} \\
\end{array}$ & $\begin{array}{c}\text { Fat } \\
\text { (grams) }\end{array}$ & $\begin{array}{c}\text { Carbohydrates } \\
\text { (grams) }\end{array}$ \\
\hline \multirow[t]{3}{*}{ Vegetables } & $\begin{array}{l}\text { Spinach, chard, mushrooms, } \\
\text { lettuce, cucumber, radish (raw) }\end{array}$ & 2 cups & & & & \\
\hline & Nopal, squash, pumpkin,tomato & 1 cup & 25 & 2 & 0 & 4 \\
\hline & $\begin{array}{l}\text { Swiss chard, cauliflower, pea, } \\
\text { broccoli, carrot }\end{array}$ & $1 / 2$ cup & & & & \\
\hline \multirow[t]{5}{*}{ Fruits } & Grapes, tejocotes & 10 pieces & & & & \\
\hline & Peach, prickly pear, (small) & 2 pieces & & & & \\
\hline & $\begin{array}{l}\text { Apple, pomegranate, orange } \\
\text { and passion fruit, (small) }\end{array}$ & 1 piece & 60 & 0 & 0 & 15 \\
\hline & Banana, pear, grapefruit & $1 / 2$ piece & & & & \\
\hline & $\begin{array}{l}\text { Pineapple, watermelon, } \\
\text { cantaloupe, strawberry, papaya }\end{array}$ & 1 cup & & & & \\
\hline \multirow[t]{4}{*}{ Cereals } & Corn or wheat tortilla (small) & 1 piece & & & & \\
\hline & $\begin{array}{l}\text { Rice, corn kernels, pasta } \\
\text { (cooked), whole grain flakes }\end{array}$ & $1 / 2$ cup & & & & \\
\hline & Whole meal rye bread or toast & 1 slice & 70 & 2 & 0 & 15 \\
\hline & Corn cob & $3 / 4$ piece & & & & \\
\hline Legumes & Bean, lentil, chickpea & $1 / 2$ cup & 120 & 8 & 1 & 20 \\
\hline \multirow{3}{*}{$\begin{array}{l}\text { Food of } \\
\text { animal } \\
\text { source }\end{array}$} & $\begin{array}{l}\text { Beef steak, chicken or turkey } \\
\text { breast, tuna }\end{array}$ & 30 grams & & & & \\
\hline & & & 75 & 7 & 5 & 0 \\
\hline & Whole egg & 1 piece & & & & \\
\hline \multirow[t]{3}{*}{$\begin{array}{l}\text { Dairy } \\
\text { products }\end{array}$} & Milk, jocoque & 1 cup & & & & \\
\hline & & & 150 & 9 & 8 & 12 \\
\hline & Natural yogurt & $3 / 4$ cup & & & & \\
\hline \multirow[t]{3}{*}{ Oils } & Avocado & $1 / 4$ piece & & & & \\
\hline & & & 45 & 0 & 5 & 0 \\
\hline & $\begin{array}{l}\text { Peanuts, sunflower and seeds } \\
\text { oils }\end{array}$ & 1 tablespoon & & & & \\
\hline
\end{tabular}

\section{Figure 1}

Numbers of individuals at each stage of study. Participants' eligibility and non-inclusion are represented according to the Los Altos University Center target population. 


\begin{tabular}{|c|c|c|c|c|c|c|}
\hline \multirow{2}{*}{$\begin{array}{c}\text { Food } \\
\text { Groups }\end{array}$} & \multirow[b]{2}{*}{ Food Items } & \multirow{2}{*}{$\begin{array}{c}\text { Standard food } \\
\text { servings }\end{array}$} & \multicolumn{4}{|c|}{ Nutritional composition } \\
\hline & & & $\begin{array}{c}\text { Energy } \\
\text { (Kcal) }\end{array}$ & $\begin{array}{l}\begin{array}{l}\text { Protein } \\
\text { (grams) }\end{array} \\
\end{array}$ & $\begin{array}{c}\text { Fat } \\
\text { (grams) }\end{array}$ & $\begin{array}{c}\begin{array}{c}\text { Carbohydrates } \\
\text { (grams) }\end{array} \\
\end{array}$ \\
\hline \multirow[t]{3}{*}{ Vegetables } & $\begin{array}{l}\text { Spinach, chard, mushrooms, } \\
\text { lettuce, cucumber, radish (raw) }\end{array}$ & 2 cups & & & & \\
\hline & Nopal, squash, pumpkin,tomato & 1 cup & 25 & 2 & 0 & 4 \\
\hline & $\begin{array}{l}\text { Swiss chard, cauliflower, pea, } \\
\text { broccoli, carrot }\end{array}$ & $1 / 2$ cup & & & & \\
\hline \multirow[t]{5}{*}{ Fruits } & Grapes, tejocotes & 10 pieces & & & & \\
\hline & Peach, prickly pear, (small) & 2 pieces & & & & \\
\hline & $\begin{array}{l}\text { Apple, pomegranate, orange } \\
\text { and passion fruit, (small) }\end{array}$ & 1 piece & 60 & 0 & 0 & 15 \\
\hline & Banana, pear, grapefruit & $1 / 2$ piece & & & & \\
\hline & $\begin{array}{l}\text { Pineapple, watermelon, } \\
\text { cantaloupe, strawberry, papaya }\end{array}$ & 1 cup & & & & \\
\hline \multirow[t]{4}{*}{ Cereals } & Corn or wheat tortilla (small) & 1 piece & & & & \\
\hline & $\begin{array}{l}\text { Rice, corn kernels, pasta } \\
\text { (cooked), whole grain flakes }\end{array}$ & $1 / 2$ cup & & & & \\
\hline & Whole meal rye bread or toast & 1 slice & 70 & 2 & 0 & 15 \\
\hline & Corn cob & $3 / 4$ piece & & & & \\
\hline Legumes & Bean, lentil, chickpea & $1 / 2$ cup & 120 & 8 & 1 & 20 \\
\hline \multirow{3}{*}{$\begin{array}{l}\text { Food of } \\
\text { animal } \\
\text { source }\end{array}$} & $\begin{array}{l}\text { Beef steak, chicken or turkey } \\
\text { breast, tuna }\end{array}$ & 30 grams & & & & \\
\hline & & & 75 & 7 & 5 & 0 \\
\hline & Whole egg & 1 piece & & & & \\
\hline \multirow[t]{3}{*}{$\begin{array}{l}\text { Dairy } \\
\text { products }\end{array}$} & Milk, jocoque & 1 cup & & & & \\
\hline & & & 150 & 9 & 8 & 12 \\
\hline & Natural yogurt & $3 / 4$ cup & & & & \\
\hline \multirow[t]{3}{*}{ Oils } & Avocado & $1 / 4$ piece & & & & \\
\hline & & & 45 & 0 & 5 & 0 \\
\hline & $\begin{array}{l}\text { Peanuts, sunflower and seeds } \\
\text { oils }\end{array}$ & 1 tablespoon & & & & \\
\hline
\end{tabular}

\section{Figure 1}

Numbers of individuals at each stage of study. Participants' eligibility and non-inclusion are represented according to the Los Altos University Center target population. 


\begin{tabular}{|c|c|c|c|c|c|c|}
\hline \multirow{2}{*}{$\begin{array}{l}\text { Food } \\
\text { Groups }\end{array}$} & \multirow[b]{2}{*}{ Food Items } & \multirow{2}{*}{$\begin{array}{l}\text { Standard food } \\
\text { servings }\end{array}$} & \multicolumn{4}{|c|}{ Nutritional composition } \\
\hline & & & $\begin{array}{c}\begin{array}{c}\text { Energy } \\
\text { (Kcal) }\end{array} \\
\end{array}$ & $\begin{array}{l}\begin{array}{l}\text { Protein } \\
\text { (grams) }\end{array} \\
\end{array}$ & $\begin{array}{c}\text { Fat } \\
\text { (grams) }\end{array}$ & $\begin{array}{c}\text { Carbohydrates } \\
\text { (grams) }\end{array}$ \\
\hline \multirow[t]{3}{*}{ Vegetables } & $\begin{array}{l}\text { Spinach, chard, mushrooms, } \\
\text { lettuce, cucumber, radish (raw) }\end{array}$ & 2 cups & & & & \\
\hline & Nopal, squash, pumpkin,tomato & 1 cup & 25 & 2 & 0 & 4 \\
\hline & $\begin{array}{l}\text { Swiss chard, cauliflower, pea, } \\
\text { broccoli, carrot }\end{array}$ & $1 / 2$ cup & & & & \\
\hline \multirow[t]{5}{*}{ Fruits } & Grapes, tejocotes & 10 pieces & & & & \\
\hline & Peach, prickly pear, (small) & 2 pieces & & & & \\
\hline & $\begin{array}{l}\text { Apple, pomegranate, orange } \\
\text { and passion fruit, (small) }\end{array}$ & 1 piece & 60 & 0 & 0 & 15 \\
\hline & Banana, pear, grapefruit & $1 / 2$ piece & & & & \\
\hline & $\begin{array}{l}\text { Pineapple, watermelon, } \\
\text { cantaloupe, strawberry, papaya }\end{array}$ & 1 cup & & & & \\
\hline \multirow[t]{4}{*}{ Cereals } & Corn or wheat tortilla (small) & 1 piece & & & & \\
\hline & $\begin{array}{l}\text { Rice, corn kernels, pasta } \\
\text { (cooked), whole grain flakes }\end{array}$ & $1 / 2$ cup & & & & \\
\hline & Whole meal rye bread or toast & 1 slice & 70 & 2 & 0 & 15 \\
\hline & Corn cob & $3 / 4$ piece & & & & \\
\hline Legumes & Bean, lentil, chickpea & $1 / 2$ cup & 120 & 8 & 1 & 20 \\
\hline \multirow{3}{*}{$\begin{array}{l}\text { Food of } \\
\text { animal } \\
\text { source }\end{array}$} & $\begin{array}{l}\text { Beef steak, chicken or turkey } \\
\text { breast, tuna }\end{array}$ & 30 grams & & & & \\
\hline & & & 75 & 7 & 5 & 0 \\
\hline & Whole egg & 1 piece & & & & \\
\hline \multirow[t]{3}{*}{$\begin{array}{l}\text { Dairy } \\
\text { products }\end{array}$} & Milk, jocoque & 1 cup & & & & \\
\hline & & & 150 & 9 & 8 & 12 \\
\hline & Natural yogurt & $3 / 4$ cup & & & & \\
\hline \multirow[t]{3}{*}{ Oils } & Avocado & $1 / 4$ piece & & & & \\
\hline & & & 45 & 0 & 5 & 0 \\
\hline & $\begin{array}{l}\text { Peanuts, sunflower and seeds } \\
\text { oils }\end{array}$ & 1 tablespoon & & & & \\
\hline
\end{tabular}

\section{Figure 1}

Numbers of individuals at each stage of study. Participants' eligibility and non-inclusion are represented according to the Los Altos University Center target population. 


\begin{tabular}{|c|c|c|c|c|c|c|}
\hline \multirow{2}{*}{$\begin{array}{c}\text { Food } \\
\text { Groups }\end{array}$} & \multirow[b]{2}{*}{ Food Items } & \multirow{2}{*}{$\begin{array}{c}\text { Standard food } \\
\text { servings }\end{array}$} & \multicolumn{4}{|c|}{ Nutritional composition } \\
\hline & & & $\begin{array}{c}\text { Energy } \\
\text { (Kcal) }\end{array}$ & $\begin{array}{l}\begin{array}{l}\text { Protein } \\
\text { (grams) }\end{array} \\
\end{array}$ & $\begin{array}{c}\text { Fat } \\
\text { (grams) }\end{array}$ & $\begin{array}{c}\begin{array}{c}\text { Carbohydrates } \\
\text { (grams) }\end{array} \\
\end{array}$ \\
\hline \multirow[t]{3}{*}{ Vegetables } & $\begin{array}{l}\text { Spinach, chard, mushrooms, } \\
\text { lettuce, cucumber, radish (raw) }\end{array}$ & 2 cups & & & & \\
\hline & Nopal, squash, pumpkin,tomato & 1 cup & 25 & 2 & 0 & 4 \\
\hline & $\begin{array}{l}\text { Swiss chard, cauliflower, pea, } \\
\text { broccoli, carrot }\end{array}$ & $1 / 2$ cup & & & & \\
\hline \multirow[t]{5}{*}{ Fruits } & Grapes, tejocotes & 10 pieces & & & & \\
\hline & Peach, prickly pear, (small) & 2 pieces & & & & \\
\hline & $\begin{array}{l}\text { Apple, pomegranate, orange } \\
\text { and passion fruit, (small) }\end{array}$ & 1 piece & 60 & 0 & 0 & 15 \\
\hline & Banana, pear, grapefruit & $1 / 2$ piece & & & & \\
\hline & $\begin{array}{l}\text { Pineapple, watermelon, } \\
\text { cantaloupe, strawberry, papaya }\end{array}$ & 1 cup & & & & \\
\hline \multirow[t]{4}{*}{ Cereals } & Corn or wheat tortilla (small) & 1 piece & & & & \\
\hline & $\begin{array}{l}\text { Rice, corn kernels, pasta } \\
\text { (cooked), whole grain flakes }\end{array}$ & $1 / 2$ cup & & & & \\
\hline & Whole meal rye bread or toast & 1 slice & 70 & 2 & 0 & 15 \\
\hline & Corn cob & $3 / 4$ piece & & & & \\
\hline Legumes & Bean, lentil, chickpea & $1 / 2$ cup & 120 & 8 & 1 & 20 \\
\hline \multirow{3}{*}{$\begin{array}{l}\text { Food of } \\
\text { animal } \\
\text { source }\end{array}$} & $\begin{array}{l}\text { Beef steak, chicken or turkey } \\
\text { breast, tuna }\end{array}$ & 30 grams & & & & \\
\hline & & & 75 & 7 & 5 & 0 \\
\hline & Whole egg & 1 piece & & & & \\
\hline \multirow[t]{3}{*}{$\begin{array}{l}\text { Dairy } \\
\text { products }\end{array}$} & Milk, jocoque & 1 cup & & & & \\
\hline & & & 150 & 9 & 8 & 12 \\
\hline & Natural yogurt & $3 / 4$ cup & & & & \\
\hline \multirow[t]{3}{*}{ Oils } & Avocado & $1 / 4$ piece & & & & \\
\hline & & & 45 & 0 & 5 & 0 \\
\hline & $\begin{array}{l}\text { Peanuts, sunflower and seeds } \\
\text { oils }\end{array}$ & 1 tablespoon & & & & \\
\hline
\end{tabular}

\section{Figure 2}

Participants' locality distribution from the Los Altos, Jalisco region. Participants' municipalities from the Los Altos Jalisco including South and North region. Other municipalities of the participants include Jesus Maria, Encarnacion de Diaz, and Teocaltiche. 


\begin{tabular}{|c|c|c|c|c|c|c|}
\hline \multirow{2}{*}{$\begin{array}{c}\text { Food } \\
\text { Groups }\end{array}$} & \multirow[b]{2}{*}{ Food Items } & \multirow{2}{*}{$\begin{array}{c}\text { Standard food } \\
\text { servings }\end{array}$} & \multicolumn{4}{|c|}{ Nutritional composition } \\
\hline & & & $\begin{array}{c}\text { Energy } \\
\text { (Kcal) }\end{array}$ & $\begin{array}{l}\begin{array}{l}\text { Protein } \\
\text { (grams) }\end{array} \\
\end{array}$ & $\begin{array}{c}\text { Fat } \\
\text { (grams) }\end{array}$ & $\begin{array}{c}\begin{array}{c}\text { Carbohydrates } \\
\text { (grams) }\end{array} \\
\end{array}$ \\
\hline \multirow[t]{3}{*}{ Vegetables } & $\begin{array}{l}\text { Spinach, chard, mushrooms, } \\
\text { lettuce, cucumber, radish (raw) }\end{array}$ & 2 cups & & & & \\
\hline & Nopal, squash, pumpkin,tomato & 1 cup & 25 & 2 & 0 & 4 \\
\hline & $\begin{array}{l}\text { Swiss chard, cauliflower, pea, } \\
\text { broccoli, carrot }\end{array}$ & $1 / 2$ cup & & & & \\
\hline \multirow[t]{5}{*}{ Fruits } & Grapes, tejocotes & 10 pieces & & & & \\
\hline & Peach, prickly pear, (small) & 2 pieces & & & & \\
\hline & $\begin{array}{l}\text { Apple, pomegranate, orange } \\
\text { and passion fruit, (small) }\end{array}$ & 1 piece & 60 & 0 & 0 & 15 \\
\hline & Banana, pear, grapefruit & $1 / 2$ piece & & & & \\
\hline & $\begin{array}{l}\text { Pineapple, watermelon, } \\
\text { cantaloupe, strawberry, papaya }\end{array}$ & 1 cup & & & & \\
\hline \multirow[t]{4}{*}{ Cereals } & Corn or wheat tortilla (small) & 1 piece & & & & \\
\hline & $\begin{array}{l}\text { Rice, corn kernels, pasta } \\
\text { (cooked), whole grain flakes }\end{array}$ & $1 / 2$ cup & & & & \\
\hline & Whole meal rye bread or toast & 1 slice & 70 & 2 & 0 & 15 \\
\hline & Corn cob & $3 / 4$ piece & & & & \\
\hline Legumes & Bean, lentil, chickpea & $1 / 2$ cup & 120 & 8 & 1 & 20 \\
\hline \multirow{3}{*}{$\begin{array}{l}\text { Food of } \\
\text { animal } \\
\text { source }\end{array}$} & $\begin{array}{l}\text { Beef steak, chicken or turkey } \\
\text { breast, tuna }\end{array}$ & 30 grams & & & & \\
\hline & & & 75 & 7 & 5 & 0 \\
\hline & Whole egg & 1 piece & & & & \\
\hline \multirow[t]{3}{*}{$\begin{array}{l}\text { Dairy } \\
\text { products }\end{array}$} & Milk, jocoque & 1 cup & & & & \\
\hline & & & 150 & 9 & 8 & 12 \\
\hline & Natural yogurt & $3 / 4$ cup & & & & \\
\hline \multirow[t]{3}{*}{ Oils } & Avocado & $1 / 4$ piece & & & & \\
\hline & & & 45 & 0 & 5 & 0 \\
\hline & $\begin{array}{l}\text { Peanuts, sunflower and seeds } \\
\text { oils }\end{array}$ & 1 tablespoon & & & & \\
\hline
\end{tabular}

\section{Figure 3}

Food grouping by the Mexican Food Equivalent System. The food items represent the main components from the traditional Mexican diet according to the Mexican Food Equivalent System. 


\begin{tabular}{|c|c|c|c|c|c|c|}
\hline \multirow{2}{*}{$\begin{array}{c}\text { Food } \\
\text { Groups }\end{array}$} & \multirow[b]{2}{*}{ Food Items } & \multirow{2}{*}{$\begin{array}{c}\text { Standard food } \\
\text { servings }\end{array}$} & \multicolumn{4}{|c|}{ Nutritional composition } \\
\hline & & & $\begin{array}{c}\begin{array}{c}\text { Energy } \\
\text { (Kcal) }\end{array} \\
\end{array}$ & $\begin{array}{l}\begin{array}{l}\text { Protein } \\
\text { (grams) }\end{array} \\
\text { (rat }\end{array}$ & $\begin{array}{c}\text { Fat } \\
\text { (grams) }\end{array}$ & $\begin{array}{c}\text { Carbohydrates } \\
\text { (grams) }\end{array}$ \\
\hline \multirow[t]{3}{*}{ Vegetables } & $\begin{array}{l}\text { Spinach, chard, mushrooms, } \\
\text { lettuce, cucumber, radish (raw) }\end{array}$ & 2 cups & & & & \\
\hline & Nopal, squash, pumpkin,tomato & 1 cup & 25 & 2 & 0 & 4 \\
\hline & $\begin{array}{l}\text { Swiss chard, cauliflower, pea, } \\
\text { broccoli, carrot }\end{array}$ & $1 / 2$ cup & & & & \\
\hline \multirow[t]{5}{*}{ Fruits } & Grapes, tejocotes & 10 pieces & & & & \\
\hline & Peach, prickly pear, (small) & 2 pieces & & & & \\
\hline & $\begin{array}{l}\text { Apple, pomegranate, orange } \\
\text { and passion fruit, (small) }\end{array}$ & 1 piece & 60 & 0 & 0 & 15 \\
\hline & Banana, pear, grapefruit & $1 / 2$ piece & & & & \\
\hline & $\begin{array}{l}\text { Pineapple, watermelon, } \\
\text { cantaloupe, strawberry, papaya }\end{array}$ & 1 cup & & & & \\
\hline \multirow[t]{4}{*}{ Cereals } & Corn or wheat tortilla (small) & 1 piece & & & & \\
\hline & $\begin{array}{l}\text { Rice, corn kernels, pasta } \\
\text { (cooked), whole grain flakes }\end{array}$ & $1 / 2$ cup & & & & \\
\hline & Whole meal rye bread or toast & 1 slice & 70 & 2 & 0 & 15 \\
\hline & Corn cob & $3 / 4$ piece & & & & \\
\hline Legumes & Bean, lentil, chickpea & $1 / 2$ cup & 120 & 8 & 1 & 20 \\
\hline \multirow[t]{3}{*}{$\begin{array}{l}\text { Food of } \\
\text { animal } \\
\text { source }\end{array}$} & $\begin{array}{l}\text { Beef steak, chicken or turkey } \\
\text { breast, tuna }\end{array}$ & 30 grams & & & & \\
\hline & & & 75 & 7 & 5 & 0 \\
\hline & Whole egg & 1 piece & & & & \\
\hline \multirow[t]{3}{*}{$\begin{array}{l}\text { Dairy } \\
\text { products }\end{array}$} & Milk, jocoque & 1 cup & & & & \\
\hline & & & 150 & 9 & 8 & 12 \\
\hline & Natural yogurt & $3 / 4$ cup & & & & \\
\hline \multirow[t]{2}{*}{ Oils } & Avocado & $1 / 4$ piece & & & & \\
\hline & $\begin{array}{l}\text { Peanuts, sunflower and seeds } \\
\text { oils }\end{array}$ & 1 tablespoon & 45 & 0 & 5 & 0 \\
\hline
\end{tabular}

\section{Figure 4}

Risk factors to Hypoadiponectinemia. Odds Ratio (OR) estimation to hypoadiponectinemia. Hypertriglyceridemia was defined as triglycerides $\geq 150 \mathrm{mg} / \mathrm{dL}$; Low HDL-c was defined at levels $<40 \mathrm{mg} / \mathrm{dL}$ in men, and $<50 \mathrm{mg} / \mathrm{dL}$ in women; abdominal obesity was determined by waist circumference $>90 \mathrm{~cm}$ in men, and $>80 \mathrm{~cm}$ in women; high LDL-c was defined as $\geq 130 \mathrm{mg} / \mathrm{dL}$; hypercholesterolemia was defined as total cholesterol $\geq 200 \mathrm{mg} / \mathrm{dL}$; insulin resistance was defined as HOMA-IR index $\geq 2.5$ [33]; LDL-c, low-density lipoprotein; VLDL-c, very low-density lipoprotein; HDL-c, high-density lipoprotein. 95\% IC, interval confidence, ${ }^{\star} p<0.05$.

\section{Supplementary Files}


This is a list of supplementary files associated with this preprint. Click to download.

- Manuscript.pdf 